\title{
Low order stabilized finite element for the full Biot formulation in Soil Mechanics at Finite Strain
}

\author{
Lluís Monforte $^{1}$ | Pedro Navas*1 | Josep Maria Carbonell ${ }^{2}$ | Marcos Arroyo $^{1}$ | Antonio Gens ${ }^{1}$
}

\author{
${ }^{1}$ Department of Geotechnical Engineering, \\ Universitat Politècnica de Catalunya - \\ BarcelonaTech, Campus Nord, Carrer Jordi \\ Girona, 1-3, 08034 Barcelona, Spain \\ ${ }^{2}$ International Center for Numerical \\ Methods in Engineering (CIMNE),Campus \\ Nord, Carrer Jordi Girona, 1-3, 08034 \\ Barcelona, Spain \\ Correspondence \\ * Pedro Navas. Email: \\ penavasal@hotmail.com
}

\begin{abstract}
Summary
This article presents a novel finite element formulation for the Biot equation using low-order elements. Additionally, an extra degree of freedom is introduced to treat the volumetric locking steaming from the effective response of the medium; its balance equation is also stabilized. The accuracy of the proposed formulation is demonstrated by means of numerical analyses.
\end{abstract}

KEYWORDS:

Poromechanics, Full-Biot, Low-Order Stabilization techniques, Large strains, Consolidation

\section{1 | INTRODUCTION}

The mechanics of fully saturated porous media is relevant for many engineering applications. A fundamental aspect of that mechanics is the description of couplings between the solid and fluid phases, for which several theoretical models have been

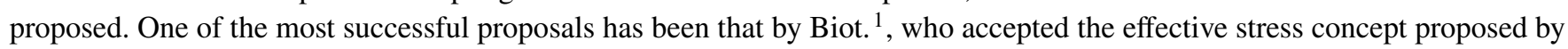
Terzaghi, and extended its reach from quasi-static one-dimensional problems to a dynamic three-dimensional setting.

The Biot equations allow for several choices of primary variables. For solutions using the finite element method, Zienkiewicz et al ${ }^{2[3]}$ discuss alternative formulations. Some of these, denoted as $\boldsymbol{u}-\dot{\boldsymbol{w}}-p_{w}, \boldsymbol{u}-\boldsymbol{U}$ or $\boldsymbol{u}-\boldsymbol{U}-p_{w}$ are able to represent the whole set of Biot equations (full Biot). Another, known as the $\boldsymbol{u}-p_{w}$ or reduced formulation is valid only when fluid accelerations are far smaller than those of the solid phase. Of those variables $\boldsymbol{u}$ and $\boldsymbol{U}$ represent, respectively, the absolute displacement vectors of the solid skeleton and the fluid phase, $\boldsymbol{w}$ the relative displacement between the phases and $p_{w}$ the fluid pressure.

The reduced $\boldsymbol{u}-p_{w}$ formulation- is not applicable to high-frequency loading, such as that resulting from impact events ${ }^{3}$. On the other hand, it is used quite often, since many seismic applications fall within its remit and the formulation is simpler and, having less degrees of freedom per node, computationally less demanding. This widespread use has brought much attention to potential numerical problems that may arise during its solution and, particularly, the development of stable formulations.

Unstable elements manifest themselves in the numerical solution as over-stiffening of mechanical response and spurious high spatial variability of the water pressure field, eventually leading to instability, non-uniqueness and mesh-dependence of the solutions. This pathology appears when the problem conditions lead to incompressibility. For water-saturated soils this is approximately the case in undrained conditions. The system of discretized equations for the $\mathbf{u}-p_{w}$ formulation, has then similar structure to that found when using a mixed $\mathbf{u}-p$ formulation of Solid Mechanics problems ${ }^{4}$. As the problem approaches zero permeability and given the quasi-incompressilibity of the soil and water constituents, it may become ill-posed from a mathematical point of view.

This numerical pathology is due to an improper finite-dimensional space in the finite element discretization and may be identified using analytical tools such as the inf-sup condition ${ }^{[5]}$ or the patch-test. More importantly, it can be also avoided or

${ }^{0}$ Abbreviations: DME, Divergence of the Momentum Equation; PPP, Polynomial Pressure Projection; FiC, Finite Calculus 
alleviated using a variety of numerical strategies. Typically, those strategies have been first applied in single-phase fluid or solid mechanics problems and later adapted to the coupled hydromechanical case.

An example is Finite Increment Calculus (FIC) which was first developed by Oñate to address to Fluid Dynamics and Solid Mechanics problems ${ }^{677}$ and later applied to the $\boldsymbol{u}-p_{w}$ formulation ${ }^{8[9}$. In this method, balance equations are expressed in a space/time domain of finite size and higher-order terms in the Taylor series expansion are retained. The FIC form of the balance equations contains derivatives of the classical differential equations multiplied by characteristic distances in space and/or time. This approach adds significant complexity to the finite element discretization procedures.

Stable formulations may be also achieved with fractional step algorithms (split-operator methods); equations are solved in a staggered fashion and the time integration algorithm provides stabilization ${ }^{4}$. This approach requires semi-explicit integration steps that may significantly increase the computational burden.

Finally, the spurious modes that appear due to the failure to satisfy the inf-sup condition can be also addressed using simpler, single-step monolithic solvers. Two main strategies are still possible: either to use more complex, but stable, finite elements with different order interpolation of displacement and water pressure fields, or to apply stabilization procedures to originally unstable finite elements ${ }^{10}$. Equal-order elements are preferable for various reasons. Numerical implementation is simpler when the same shape functions are used for all variables ${ }^{11}$. Additionally, low order elements are preferred for its flexibility in adaptive remeshing codes such as the Particle Finite Element method, which is typically reliant on linear elements 12 13/14.

A variety of techniques have been employed for low-order finite element stabilization. Hafez and Soliman ${ }^{15}$ proposed to add to the mass balance equation the divergence of the momentum equation; this technique was applied to the $\boldsymbol{u}-p_{w}$ formulation by Pastor et al ${ }^{10}$. Another technique is the Polynomial projection technique (PPP) ${ }^{16}$, that adds a term proportional to the projection of the pressure in a lower order shape function. This technique was successfully applied to the $\boldsymbol{u}-p_{w}$ first at small strains by White y Borja ${ }^{17}$ and later at finite strains by Sun et al. 18 .

Apart from undrained conditions, incompressibility may also arise in soil mechanics simply from the mechanical response of the solid phase. This happens, for instance, when failure is reached in Critical State soil models. As noted in the literature $\frac{19118}{}$, this response may lead to volumetric locking. Separate numerical techniques to treat this extra source of incompressibility are required, and they have been addressed in models using the reduced $\boldsymbol{u}-p_{w}$ formulation. Sun et a ${ }^{[1820}$ proposed a formulation with an assumed deformation gradient, that has the F-bar method as a limiting case. Monforte et al ${ }^{21}$ examined three-field mixed formulations in which either the effective pressure or the Jacobian were added as supplementary nodal variables and stabilization techniques were employed in all scalar equations.

In contrast to the extensive work done with the reduced formulation, the development of equal-order stable formulations and the consequent need for stabilization has received much less attention for the full Biot case. Jeremić et al ${ }^{[22}$ employed a $\boldsymbol{u}-\boldsymbol{U}-p_{w}$ formulation where the pore pressure variable was added for stabilization purposes. They addressed elasto-plastic problems with a small strain formulation. They used elements with different interpolation order for pore pressure and other nodal variables and did not note any spurious mode, although the focus of their work was mostly on seismic applications.

Markert et al ${ }^{233}$ presented a systematic comparison of different numerical approaches to the full set of poroelastic small strain equations. They explored high order elements, in which the mixture motion variables were described with second order interpolants and first order shape functions were used for the fluid variables (relative velocity and pressure). They compared with simpler elements in which linear interpolants were used for all the variables. They found that in low permeability cases equal order interpolants yielded an inaccurate displacement solution and a completely wrong pore pressure field. They explored an split-operator stabilization technique for the equal order elements, as well as a variety of time integration rules.

Sabetamal et al ${ }^{2425}$ studied the impact of objects into the soil using a $\boldsymbol{u}-\dot{\boldsymbol{w}}-p_{w}$ formulation. An arbitrary LagrangianEulerian method with high order elements was used for the solution. The field equations were extended to large strain through an hypoelastic approach. Finally, it is worth mentioning that Navas et al ${ }^{26 / 27}$ also presented a numerical solution for the full Biot equations at large strains, using the $\boldsymbol{u}-\boldsymbol{w}$ formulation. The equations were solved using a meshfree method in which the shape functions are based on the principle of Local Maximum Entropy.

This work presents a methodology to solve the full Biot poroelastic equations at large strains using a finite element mesh with low order elements. The motivation lies in extending the range of geotechnical applications of the Particle Finite Element Method (PFEM) to cover high frequency problems, such as impact loading. PFEM was originally developed to address fluid-structure

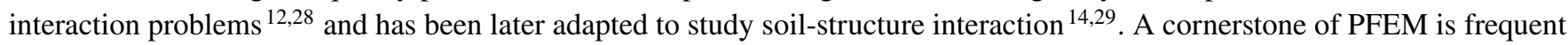
re-meshing for which low order elements are very advantageous. The elements employed have equal order interpolation for all the nodal basic variables, chosen as the mixture displacement, water relative displacement and water pressure, a formulation with shorthand $\boldsymbol{u}-\boldsymbol{w}-p_{w}$, resulting in a P1-P1-P1 element. 
The focus of this work lies on the stabilization techniques that are incorporated in the solution to avoid pore pressure spurious oscillations. Two techniques, namely polynomial pressure projection (PPP) and divergence of momentum (DME), are implemented and tested. They both allow for a monolithic (i.e. non-staggered) solution of the field equations. Apart from that, and to mitigate the volumetric locking that may arise from the solid phase response a mixed stabilized formulation previously applied to the simplified $\mathbf{u}-p_{w}$ formulation ${ }^{21}$ is extended to the dynamic range.

This paper is structured as follows: first, the field equations are presented. The equations are then discretized in space by Finite Elements and in time using a Newmark scheme; a consistent linearization scheme is presented for numerical integration. The applied stabilization techniques are then presented in detail, developing a new formula to bound the stabilization parameter in the PPP stabilizing term. The performance of the developed numerical scheme is assessed with 1-D and 2-D numerical analyses of some representative poroelastodynamic problems. Finally, a number of conclusions are drawn.

\section{2 | MATHEMATICAL MODEL}

\subsection{Strong form of the balance equations}

Let $\boldsymbol{v}$ and $\boldsymbol{v}^{w}$ represent, respectively, the absolute velocity vectors of the solid skeleton and the fluid phase. The relative velocity of the fluid phase with respect to the solid one, $\dot{\boldsymbol{w}}$, is expressed as

$$
\dot{\boldsymbol{w}}=n\left(\boldsymbol{v}^{w}-\boldsymbol{v}\right),
$$

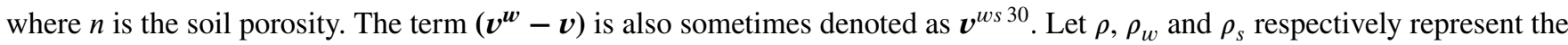
mixture, fluid phase and solid particle densities, related through:

$$
\rho=n \rho_{w}+(1-n) \rho_{s} .
$$

whereas the porosity is related to the initial porosity, $n_{0}$, and the determinant of the deformation gradient, $J$, through:

$$
n=1-\frac{1-n_{0}}{J}
$$

The mass balance equation for fluid-saturated porous media with compressible solid grains and water phase may be expressed as 30 :

$$
\operatorname{div}(\dot{\boldsymbol{u}})+\operatorname{div}(\dot{\boldsymbol{w}})+\frac{1}{Q}\left[\dot{p}_{w}+\operatorname{grad} p_{w} \cdot \frac{\dot{\boldsymbol{w}}}{n}\right]=0
$$

where $p_{w}$ is the Cauchy water pressure, $\dot{\boldsymbol{u}}=\frac{\mathrm{d} \boldsymbol{u}}{\mathrm{d} t}$ and $\dot{\boldsymbol{w}}=\frac{\mathrm{d} \boldsymbol{w}}{\mathrm{d} t}$ are the material time derivatives with respect to the solid phase of $\boldsymbol{u}$ and $\boldsymbol{w} \cdot Q$ stands for the Biot modulus or mixture compressibility modulus, related to the solid and liquid phase compressibility, $K_{s}$ and $K_{w}$ through:

$$
Q=\left[\frac{1-n}{K_{s}}+\frac{n}{K_{w}}\right]^{-1}
$$

The linear momentum balance equation for the mixture reads:

$$
\operatorname{div}[\boldsymbol{\sigma}]-\rho \ddot{\boldsymbol{u}}-\rho_{w}(\ddot{\boldsymbol{w}}+\operatorname{grad} \dot{\boldsymbol{U}} \cdot \dot{\boldsymbol{w}})+\rho \boldsymbol{g}=\mathbf{0}
$$

Finally, the relative velocity of the fluid, $\dot{\boldsymbol{w}}$, is constrained by Darcy's law as 3

$$
\dot{\boldsymbol{w}}=n \boldsymbol{v}^{w s}=\boldsymbol{k} \cdot\left[-\operatorname{grad} p_{w}+\rho_{w}\left(\boldsymbol{g}-\ddot{\boldsymbol{u}}-\boldsymbol{a}^{w s}\right)\right]
$$

where $\boldsymbol{g}$ represents gravity, $\boldsymbol{v}^{w s}$ stands for the relative water velocity with respect to the solid phase, $\ddot{\boldsymbol{u}}$ and $\boldsymbol{a}^{w s}$ are the solid acceleration and the relative water acceleration with respect to the solid respectively, $\boldsymbol{k}=k \boldsymbol{I}$ is the permeability tensor, that is further related to the intrinsic permeability, $k^{\prime}$ expressed in $\left[\mathrm{m}^{2}\right]$ and the hydraulic conductivity, $\kappa[\mathrm{m} / \mathrm{s}]$, by the following equation

$$
k=\frac{k^{\prime}}{\mu_{w}}=\frac{\kappa}{\rho_{w} g} .
$$

where $\mu_{w}$ is the fluid viscosity.

Taking into account the definition of the fluid acceleration, the time derivative of the relative water velocity:

$$
\boldsymbol{a}^{w s}=\dot{\boldsymbol{v}}^{w s}+\operatorname{grad}\left(\dot{\boldsymbol{u}}+\boldsymbol{v}^{w s}\right) \cdot \boldsymbol{v}^{w s}
$$


the following expression for the Darcy's equation, Equation (7), may be obtained:

$$
-\operatorname{grad} p_{w}-\boldsymbol{k}^{-1} \cdot \dot{\boldsymbol{w}}+\rho_{w}\left(\boldsymbol{g}-\ddot{\boldsymbol{u}}-\frac{\ddot{\boldsymbol{w}}+\operatorname{grad} \dot{\boldsymbol{U}} \cdot \dot{\boldsymbol{w}}}{n}\right)=\mathbf{0}
$$

According to the principle of effective stress, the total stress tensor, $\sigma$, is equal to the sum of the pore pressure, $p_{w}$, and the effective stress, $\sigma^{\prime}$ :

$$
\sigma=\sigma^{\prime}-p_{w} \mathbf{I}
$$

where $\sigma^{\prime}=\hat{\sigma}^{\prime}(\boldsymbol{F}, V)$ is the effective Cauchy stress tensors (positive in tension), that depends on the constitutive model through the deformation gradient, $\boldsymbol{F}$, and a set of internal variables of the model, $V$.

The previously presented balance equations, Equations (4), (10) and (6), include some terms that are generally omitted ${ }^{3033}$. For instance, the last term of Equation (4), $\frac{1}{Q} \operatorname{grad} p_{w} \cdot \frac{\dot{\boldsymbol{w}}}{n}$, stems from the convective derivative (with respect to the solid phase) of the change of water density; this effect may be assumed negligible for soil mechanics applications ${ }^{3}$. Keeping these terms in the overall numerical formulation would just add some extra terms to the finite element mass and damping matrices; this more complete model would also suffer from the same kind of instabilities than appear in the formulation without convective terms and it is presumed that the stabilizing solutions developed here would also be benefitial in that case. As such, the balance equations can be simplified to:

$$
\begin{cases}\operatorname{div}\left[\boldsymbol{\sigma}^{\prime}-p_{w} \mathbf{I}\right]-\rho \ddot{\boldsymbol{u}}-\rho_{w} \ddot{\boldsymbol{w}}+\rho \mathbf{g}=\mathbf{0} & \\ -\operatorname{grad} p_{w}-\boldsymbol{k}^{-1} \cdot \dot{\boldsymbol{w}}+\rho_{w}\left(\boldsymbol{g}-\ddot{\boldsymbol{u}}-\frac{\ddot{\boldsymbol{w}}}{n}\right)=\mathbf{0} & \\ \operatorname{div}(\dot{\boldsymbol{u}})+\operatorname{div}(\dot{\boldsymbol{w}})+\frac{1}{Q} \dot{p}_{w}=0 & \text { in } \Gamma_{u} \\ \boldsymbol{u}=\overline{\boldsymbol{u}} & \text { in } \Gamma_{t} \\ \boldsymbol{\sigma} \cdot \boldsymbol{n}=\overline{\boldsymbol{t}} & \text { in } \Gamma_{w} \\ \boldsymbol{w}=\overline{\boldsymbol{w}} & \text { in } \Gamma_{p_{w}} \\ p_{w}=\bar{p}_{w} & \end{cases}
$$

where $\Gamma_{u}$ and $\Gamma_{t}$ defines the boundary of the domain where displacements $\overline{\boldsymbol{u}}$ and tractions $\overline{\boldsymbol{t}}$ are prescribed. Water pressure $\bar{p}_{w}$ and water velocity $\overline{\boldsymbol{w}}$ are imposed at $\Gamma_{p_{w}}$ and $\Gamma_{w}$.

\section{2 | Discretized system of equations}

This subsection presents the numerical treatment of the balance equations. First, the weak form of the balance equations is obtained to introduce the finite element spatial discretization. The semi-discrete equations are then temporally integrated with a Generalized-Newmark scheme.

\subsection{1 | Weak form and spatial discretization}

The derivation of the weak form of the problem defined by Equations (12) starts by multiplying the local balance equations by tests functions (being $\delta \boldsymbol{u}, \delta \boldsymbol{w}$ and $\delta p_{w}$ the virtual displacement of the solid and the fluid phase and the virtual pore pressure respectively) and integrating over the domain. For the linear momentum balance equation this procedure gives:

$$
\int_{\Omega} \delta \boldsymbol{u} \cdot\left[\operatorname{div}(\boldsymbol{\sigma})-\rho \ddot{\boldsymbol{u}}-\rho_{w} \ddot{\boldsymbol{w}}+\rho \boldsymbol{g}\right] \mathrm{d} \Omega=0
$$

Applying Green's Theorem, Equation (13) yields:

$$
\int_{\Omega} \delta \boldsymbol{u} \cdot\left[-\rho \ddot{\boldsymbol{u}}-\rho_{w} \ddot{\boldsymbol{w}}+\rho \boldsymbol{g}\right] \mathrm{d} \Omega-\int_{\Omega} \boldsymbol{\sigma}: \operatorname{grad} \delta \boldsymbol{u} \mathrm{d} \Omega+\int_{\Gamma_{t}} \overline{\boldsymbol{t}} \cdot \delta \boldsymbol{u} \mathrm{d} \gamma=0
$$

This equation can be rearranged taking into account Terzaghi's principle of effective stress:

$$
\int_{\Omega} \delta \boldsymbol{u} \cdot\left[-\rho \ddot{\boldsymbol{u}}-\rho_{w} \ddot{\boldsymbol{w}}+\rho \boldsymbol{g}\right] \mathrm{d} \Omega-\int_{\Omega} \boldsymbol{\sigma}^{\prime}: \operatorname{grad} \delta \boldsymbol{u} \mathrm{d} \Omega+\int_{\Omega} p_{w} \operatorname{div} \delta \boldsymbol{u} \mathrm{d} \Omega+\int_{\Gamma_{t}} \overline{\boldsymbol{t}} \cdot \delta \boldsymbol{u} \mathrm{d} \gamma=0 \quad \forall \delta \boldsymbol{u} \in \mathcal{T}_{u}(\Omega)
$$


The case of the fluid linear balance equation is seen following:

$$
-\int_{\Omega} \operatorname{grad}\left(p_{w}\right) \cdot \delta \boldsymbol{w} \mathrm{d} \Omega-\int_{\Omega} \dot{\boldsymbol{w}} \cdot \mathbf{k}^{-1} \cdot \delta \boldsymbol{w} \mathrm{d} \Omega+\int_{\Omega} \rho_{w}\left(\boldsymbol{g}-\ddot{\boldsymbol{u}}-\frac{\ddot{\boldsymbol{w}}}{n}\right) \cdot \delta \boldsymbol{w} \mathrm{d} \Omega=0 \quad \forall \delta \boldsymbol{w} \in \mathcal{T}_{w}(\Omega)
$$

Finally, the weak form of the mass conservation law yields:

$$
\int_{\Omega} \operatorname{div}(\dot{\boldsymbol{u}}) \cdot \delta p_{w} \mathrm{~d} \Omega+\int_{\Omega} \operatorname{div}(\dot{\boldsymbol{w}}) \cdot \delta p_{w} \mathrm{~d} \Omega+\int_{\Omega} \frac{\dot{p}_{w}}{Q} \cdot \delta p_{w} \mathrm{~d} \Omega=0 \quad \forall \delta p_{w} \in \mathcal{T}_{p_{w}}(\Omega)
$$

where, in Equations (15), (16) and (17), the solution fields belong to the spaces: $\boldsymbol{u} \in S_{u}(\Omega), \boldsymbol{w} \in S_{w}(\Omega)$ and $p_{w} \in S_{p_{w}}(\Omega)$, that are defined as:

$$
\begin{aligned}
& S_{u}(\Omega)=\left\{\boldsymbol{u} \mid \boldsymbol{u} \in\left[H^{1}(\Omega)\right]^{n}, \boldsymbol{u}=\overline{\boldsymbol{u}} \text { in } \Gamma_{u}\right\} \quad \mathcal{T}_{u}(\Omega)=\left\{\boldsymbol{u} \mid \boldsymbol{u} \in\left[H^{1}(\Omega)\right]^{n}, \boldsymbol{u}=\mathbf{0} \text { in } \Gamma_{u}\right\} \\
& S_{w}(\Omega)=\left\{\boldsymbol{w} \mid \boldsymbol{w} \in H(\operatorname{div}, \Omega), \boldsymbol{w}=\overline{\boldsymbol{w}} \text { in } \Gamma_{w}\right\} \quad \mathcal{T}_{w}(\Omega)=\left\{\boldsymbol{w} \mid \boldsymbol{w} \in\left[L^{2}(\Omega)\right]^{n}, \boldsymbol{w}=\mathbf{0} \text { in } \Gamma_{w}\right\} \\
& S_{p_{w}}(\Omega)=\left\{p_{w} \mid p_{w} \in H^{1}(\Omega), \quad p_{w}=\overline{p_{w}} \text { in } \Gamma_{p_{w}}\right\} \quad \mathcal{T}_{p_{w}}(\Omega)=\left\{p_{w} \mid p_{w} \in L^{2}(\Omega), p_{w}=0 \text { in } \Gamma_{p_{w}}\right\}
\end{aligned}
$$

where $H^{1}(\Omega)$ is the space of square integrable functions whose gradients are also square integrable, $L^{2}(\Omega)$ is the space of square integrable functions whereas $H(\operatorname{div}, \Omega)$ is the space of square integrable vector functions whose divergence is also square integrable.

The derivation presented above is slightly different to that followed by previous researchers. For instance Markert et al ${ }^{23}$ first introduce the effective stress principle in the weak form of the linear momentum balance equation, Equation (13) and afterwards apply Green's Theorem only to the effective stress term; by doing this, the traction due to the effective response of the material is imposed in the Neumann part of the boundary and special procedures to impose the traction due to the total stress are required.

In this work the divergence theorem is not applied to the weak forms of the mass balance and Darcy's equations, Equations (16) and (17). This results in another difference with the work of Markert et al ${ }^{[23}$ and Sabetamal et al ${ }^{2425}$ who apply that theorem and obtain different weak forms. In particular, they arrive to the following mass balance equation 23/2425:

$$
\int_{\Omega} \operatorname{div}(\dot{\boldsymbol{u}}) \cdot \delta p_{w} \mathrm{~d} \Omega-\int_{\Omega} \dot{\boldsymbol{w}} \cdot \operatorname{grad} \delta p_{w} \mathrm{~d} \Omega+\int_{\Omega} \frac{\dot{p}_{w}}{Q} \cdot \delta p_{w} \mathrm{~d} \Omega+\int_{\Gamma_{g}} \delta p_{w}(\boldsymbol{n} \cdot \dot{\overline{\boldsymbol{w}}}) \mathrm{d} \gamma=0
$$

where the last term appears due to the application of the Divergence theorem and represents the imposed normal water flux at the boundary $\Gamma_{w}$. However, this flux is also imposed as Dirichlet boundary conditions in the Darcy's equation, Equation (16). However, it seems unnecessary to specify the normal flux boundary condition twice: as a boundary integral in the mass balance equation and as Dirichlet conditions in the Darcy's equation. Using the same rationale, it can be seen also that is not necessary to apply the Divergence theorem to Darcy's equation, Equation (16.

It is worth mentioning that applying the Divergence theorem to the first term of the fluid linear balance equation, Equation (16), the balance equations do not involve any gradient of the water pressure field 3132 . Therefore, it is no longer necessary to have a water pressure field whose gradients are square integrable. By doing so, displacements, Darcy's velocity and pore pressure may be discretized using linear triangles, lowest order Raviart-Thomas and piece-wise constant shape functions respectively, which allows for element-wise mass conservation ${ }^{3132}$. In the undrained limit, this element is unstable and exhibit pore pressure oscillations, that might be solved by employing a quadratic discretization for the skeleton displacement ${ }^{33}$.

After obtaining the weak form of the balance equations, Eqs. [15), (16) and (17), the semi-discrete equations of the hydromechanical formulation are obtained. In this work, linear and equal shape functions are used for all the field variables: solid displacement, water relative displacement and water pressure. In particular, for two-dimensional problems, three-nodded linear triangles are used, that have 5 degrees of freedom at each node. Consequently, the continuous spaces of functions of the solution and test functions, Equation 2.2.1, are discretized into the discrete functional spaces:

$$
\begin{array}{cl}
S_{u}^{h}(\Omega)=\left\{\boldsymbol{u} \mid \boldsymbol{u} \in\left[\mathcal{P}\left(\Omega, \mathcal{T}_{h}\right)\right]^{n}, \quad \boldsymbol{u}=\overline{\boldsymbol{u}} \text { in } \Gamma_{u}\right\} & \mathcal{T}_{u}^{h}(\Omega)=\left\{\boldsymbol{u} \mid \boldsymbol{u} \in\left[\mathcal{P}\left(\Omega, \mathcal{T}_{h}\right)\right]^{n}, \quad \boldsymbol{u}=\mathbf{0} \text { in } \Gamma_{u}\right\} \\
S_{w}^{h}(\Omega)=\left\{\boldsymbol{w} \mid \boldsymbol{w} \in\left[\mathcal{P}\left(\Omega, \mathcal{T}_{h}\right)\right]^{n}, \boldsymbol{w}=\overline{\boldsymbol{w}} \text { in } \Gamma_{w}\right\} & \mathcal{T}_{w}^{h}(\Omega)=\left\{\boldsymbol{w} \mid \boldsymbol{w} \in\left[\mathcal{P}\left(\Omega, \mathcal{T}_{h}\right)\right]^{n} \boldsymbol{w}=\mathbf{0} \text { in } \Gamma_{w}\right\} \\
S_{p_{w}}^{h}(\Omega)=\left\{p_{w} \mid p_{w} \in \mathcal{P}\left(\Omega, \mathcal{T}_{h}\right), \quad p_{w}=\overline{p_{w}} \text { in } \Gamma_{p_{w}}\right\} & \mathcal{T}_{p_{w}^{h}}^{h}(\Omega)=\left\{p_{w} \mid p_{w} \in \mathcal{P}\left(\Omega, \mathcal{T}_{h}\right), p_{w}=0 \text { in } \Gamma_{p_{w}}\right\}
\end{array}
$$

where $\mathcal{P}\left(\Omega, \mathcal{T}_{h}\right)=\left\{\boldsymbol{u}\left|\boldsymbol{u} \in C^{0}(\Omega), \boldsymbol{u}\right|_{\Omega_{e}} \in P_{1}\left(\Omega_{e}\right) \forall \Omega_{e} \in \mathcal{T}_{h}\right\}, P_{1}\left(\Omega_{e}\right)$ is the space of linear polynomials in $\Omega_{e}$ and $\mathcal{T}_{h}$ is a partition of $\Omega$ made of non-overlapping elements $\Omega_{e}$. 
The interpolants employed are:

$$
\left\{\begin{array}{l}
\boldsymbol{u} \approx \boldsymbol{u}^{h}=\mathbf{N}_{u} \cdot \tilde{\boldsymbol{u}} \\
\boldsymbol{w} \approx \boldsymbol{w}^{h}=\mathbf{N}_{u} \cdot \tilde{\boldsymbol{w}} \\
p_{w} \approx p_{w}^{h}=\mathbf{N} \cdot \tilde{\boldsymbol{p}}_{w}
\end{array}\right.
$$

where $\square^{h}$ represents the finite element approximation of the field $\square$ and $\tilde{\square}$ the nodal values. $\mathbf{N}=\left[N_{1}, N_{2}, \ldots, N_{m}\right]$ and $\mathbf{N}_{u}=$ $\left[N_{1} \mathbf{I}, N_{2} \mathbf{I}, \ldots, N_{m} \mathbf{I}\right]$ are the shape functions and $m$ is the number of nodes. Since the shape functions are defined in the reference configuration and time independent, $\mathbf{N}=\mathbf{N}(X)$, the following property holds: $\square^{h}=\mathbf{N} \cdot \dot{\tilde{\square}}$.

Therefore, the semi-discrete form of the hydromechanical formulation, Equations (12), is given by:

$$
\left\{\begin{array}{l}
\mathbf{f}^{i n t}\left(\boldsymbol{\sigma}^{\prime}\right)-\mathbf{Q} \cdot \tilde{\mathbf{p}}_{w}+\mathbf{M}^{s} \cdot \ddot{\tilde{\boldsymbol{u}}}+\mathbf{M}^{w} \cdot \ddot{\tilde{\boldsymbol{w}}}=\mathbf{f}_{s}^{\text {ext }} \\
\mathbf{R} \cdot \tilde{\mathbf{p}}_{w}+\mathbf{M}_{k} \cdot \dot{\tilde{\boldsymbol{w}}}+\mathbf{M}^{w} \cdot \ddot{\tilde{\boldsymbol{u}}}+\frac{1}{n} \mathbf{M}^{w} \cdot \ddot{\tilde{\boldsymbol{w}}}=\mathbf{f}_{w}^{e x t} \\
\mathbf{Q}^{T} \cdot \dot{\tilde{\mathbf{u}}}+\mathbf{Q}^{T} \cdot \dot{\tilde{\mathbf{w}}}+\frac{1}{Q} \mathbf{M}_{p} \cdot \dot{\tilde{\mathbf{p}}}_{w}=\mathbf{0}
\end{array}\right.
$$

The matrices appearing of the previous expression are given by:

$$
\begin{gathered}
\mathbf{f}^{i n t}\left(\boldsymbol{\sigma}^{\prime}\right)=\int_{\Omega} \mathbf{B}^{T} \cdot \underline{\boldsymbol{\sigma}}^{\prime} \mathrm{d} \Omega \\
\mathbf{Q}=\int_{\Omega} \mathbf{B}^{T} \cdot \mathbf{I} \cdot \mathbf{N} \mathrm{d} \Omega \\
\mathbf{R}=\int_{\Omega} \mathbf{N}_{u}^{T} \cdot \nabla \mathbf{N} \mathrm{d} \Omega \\
\mathbf{M}_{k}=\int_{\Omega} \mathbf{N}_{u}^{T} \cdot \mathbf{k}^{-1} \cdot \mathbf{N}_{u} \mathrm{~d} \Omega \\
\mathbf{M}_{p}=\int_{\Omega} \mathbf{N}^{T} \cdot \mathbf{N} \mathrm{d} \Omega \\
\mathbf{M}^{\pi}=\rho_{\pi} \int_{\Omega} \mathbf{N}_{u}^{T} \cdot \mathbf{N}_{u} \mathrm{~d} \Omega
\end{gathered}
$$

where $\mathbf{B}$ has the same form that the small deformation strain-displacement matrix ${ }^{34}$ and $\underline{\boldsymbol{\sigma}}$ represents the Voigt notation of $\boldsymbol{\sigma}$. Finally, the expressions of the external forces, $\mathbf{f}_{\pi}^{\text {ext }}$ are:

$$
\begin{gathered}
\mathbf{f}_{s}^{\text {ext }}=\int_{\Omega} \rho_{s} \mathbf{N}_{u}^{T} \cdot \mathbf{g} \mathrm{d} \Omega+\int_{\Gamma_{t}} \mathbf{N}_{u}^{T} \cdot \overline{\boldsymbol{t}}, \mathrm{d} \gamma \\
\mathbf{f}_{w}^{\text {ext }}=\int_{\Omega} \rho_{w} \mathbf{N}_{u}^{T} \cdot \mathbf{g} \mathrm{d} \Omega
\end{gathered}
$$

In matrix form, the system of equations defined by Equation (22) yields:

$$
\left[\begin{array}{ccc}
\mathbf{M}^{s} & \mathbf{M}^{w} & \mathbf{0} \\
\mathbf{M}^{w} & \frac{1}{n} \mathbf{M}^{w} & \mathbf{0} \\
\mathbf{0} & \mathbf{0} & \mathbf{0}
\end{array}\right]\left[\begin{array}{c}
\ddot{\tilde{\mathbf{u}}} \\
\ddot{\tilde{\mathbf{w}}} \\
\ddot{\tilde{\mathbf{p}}}
\end{array}\right]+\left[\begin{array}{ccc}
\mathbf{0} & \mathbf{0} & \mathbf{0} \\
\mathbf{0} & \mathbf{M}_{k} & \mathbf{0} \\
\mathbf{Q}^{T} & \mathbf{Q}^{T} & \frac{1}{Q} \mathbf{M}_{p}
\end{array}\right]\left[\begin{array}{c}
\dot{\tilde{\mathbf{u}}} \\
\dot{\tilde{\mathbf{w}}} \\
\tilde{\tilde{\mathbf{p}}}_{w}
\end{array}\right]=\left[\begin{array}{c}
\mathbf{f}_{s}^{e x t} \\
\mathbf{f}_{w}^{e x t} \\
\mathbf{0}
\end{array}\right]-\left[\begin{array}{c}
\mathbf{f}^{i n t}\left(\boldsymbol{\sigma}^{\prime}\right)-\mathbf{Q} \cdot \tilde{\mathbf{p}}_{w} \\
\mathbf{R} \cdot \tilde{\mathbf{p}}_{w} \\
\mathbf{0}
\end{array}\right]
$$

\subsection{2 | Time integration scheme}

In the $\boldsymbol{u}-\boldsymbol{w}-p_{w}$ formulation here employed, each node contains both solid and fluid displacement degrees of freedom, $\boldsymbol{u}$ and $\boldsymbol{w}$, as well as the pore pressure, $p_{w}$. That can be written as:

$$
\hat{\boldsymbol{u}}=\left[\begin{array}{lll}
\tilde{\boldsymbol{u}} & \tilde{\boldsymbol{w}} & \tilde{p}_{w}
\end{array}\right]^{T}
$$

After assembling the elementary matrices, the final system of equations 31 may be expressed as

$$
\boldsymbol{M} \ddot{\hat{\boldsymbol{u}}}_{k+1}+\boldsymbol{C} \dot{\hat{\boldsymbol{u}}}_{k+1}=\boldsymbol{P}_{k+1}-\boldsymbol{R}_{k+1}
$$


where $\boldsymbol{R}, \boldsymbol{C}$ and $\boldsymbol{M}$ respectively denote the internal forces vector and damping and mass matrices, whereas $\boldsymbol{P}$ is the external forces vector, which contains gravity and other external nodal forces. $k+1$ indicates the current step.

An implicit monolithic solver is used for time integration of Eq. (32). According to Wriggers ${ }^{35}$ time integration schemes may be generally written in terms of the incremental displacements, i.e.

$$
\begin{aligned}
& \ddot{\hat{\boldsymbol{u}}}_{k+1}=\alpha_{1} \Delta \hat{\boldsymbol{u}}_{k+1}-\alpha_{2} \dot{\hat{\boldsymbol{u}}}_{k}-\alpha_{3} \ddot{\hat{\boldsymbol{u}}}_{k} \\
& \dot{\hat{\boldsymbol{u}}}_{k+1}=\alpha_{4} \Delta \hat{\boldsymbol{u}}_{k+1}+\alpha_{5} \dot{\hat{\boldsymbol{u}}}_{k}+\alpha_{6} \ddot{\hat{\boldsymbol{u}}}_{k}
\end{aligned}
$$

For the Newmark method,, 35 these coefficients yield:

$$
\begin{gathered}
\alpha_{1}=\frac{1}{\beta \Delta t^{2}} \quad \alpha_{2}=\frac{1}{\beta \Delta t} \quad \alpha_{3}=\frac{1}{2 \beta}-1 \\
\alpha_{4}=\frac{\gamma}{\beta \Delta t} \quad \alpha_{5}=1-\frac{\gamma}{\beta} \quad \alpha_{6}=\left(1-\frac{\gamma}{2 \beta}\right) \Delta t
\end{gathered}
$$

Where $\gamma$ and $\beta$ are the traditional parameters of the Newmark method. Based on the parametric analysis of Kontoe et al. ${ }^{\text {[36, }}$ in the present work the values $\gamma=0.6$ and $\beta=0.3025$ are employed. Inserting Eqs. 33. 34) in Eq. 32), it follows that:

$$
\boldsymbol{G}_{k+1}=\boldsymbol{M}\left[\alpha_{1} \Delta \hat{\boldsymbol{u}}_{k+1}-\alpha_{2} \dot{\hat{\boldsymbol{u}}}_{k}-\alpha_{3} \ddot{\hat{\boldsymbol{u}}}_{k}\right]+\boldsymbol{C}\left[\alpha_{4} \Delta \hat{\boldsymbol{u}}_{k+1}+\alpha_{5} \dot{\hat{\boldsymbol{u}}}_{k}+\alpha_{6} \ddot{\hat{\boldsymbol{u}}}_{k}\right]+\boldsymbol{R}_{k+1}-\boldsymbol{P}_{k+1}=\mathbf{0}
$$

Any Newton method can be applied to determine the unknown displacements $\frac{35}{3}$. As known, the tangential stiffness matrix corresponds to the derivative of the internal forces, i.e.

$$
\boldsymbol{K}\left(\hat{\boldsymbol{u}}_{k+1}^{i}\right)=\boldsymbol{K}_{k+1}^{i}=\left.\frac{\partial \boldsymbol{R}}{\partial \hat{\boldsymbol{u}}}\right|_{\hat{\boldsymbol{u}}_{k+1}^{i}}
$$

Where $i$ represents the iteration index. Collecting terms the iteration scheme may be written as

$$
\begin{aligned}
{\left[\alpha_{1} \boldsymbol{M}+\alpha_{4} \boldsymbol{C}+\boldsymbol{K}_{k+1}^{i}\right] \Delta \hat{\boldsymbol{u}}_{k+1}^{i+1} } & =-\boldsymbol{G}\left(\hat{\boldsymbol{u}}_{k+1}^{i}\right) \\
\text { where } \hat{\boldsymbol{u}}_{k+1}^{i+1} & =\hat{\boldsymbol{u}}_{k+1}^{i}+\Delta \hat{\boldsymbol{u}}_{k+1}^{i+1}
\end{aligned}
$$

The iteration finishes when $\boldsymbol{G}_{k+1}^{i}$ is lower than a given tolerance.

\subsection{3 | Linearization}

In the solution of the non-linear equations, Taylor's series are employed in the current configuration. After the linearization of the deformation mapping $\chi$, Eq. 35 becomes:

$$
\boldsymbol{G}(\bar{\chi}, \boldsymbol{\eta}, \Delta \hat{\boldsymbol{u}})_{k+1}^{i+1} \cong \boldsymbol{G}(\bar{\chi}, \boldsymbol{\eta})_{k+1}^{i}+\boldsymbol{D} \boldsymbol{G}(\bar{\chi}, \boldsymbol{\eta})_{k+1}^{i} \cdot \Delta \hat{\boldsymbol{u}}_{k+1}^{i+1}=\mathbf{0}
$$

where $\bar{\chi}$ is the mapping $\quad \chi=\left[\chi^{u}, \chi^{w}, \chi^{p_{w}}\right]^{T} \quad$ defined as:

$$
\left\{\begin{array}{l}
\mathbf{x}=\chi^{u}=\chi^{u}(\mathbf{X}, t) \\
\mathbf{w}=\chi^{w}=\chi^{w}(\mathbf{X}, t) \\
p_{w}=\chi^{p_{w}}=\chi^{p_{w}}(\mathbf{X}, t)
\end{array}\right.
$$

and $\quad \boldsymbol{\eta}=\left[\delta \boldsymbol{u}, \delta \boldsymbol{w}, \delta p_{w}\right]^{T}$

Taking into account the fact that operator $\boldsymbol{G}$ is composed in three parts, the derivatives take the following form:

$$
D \boldsymbol{G} \cdot \Delta \hat{\boldsymbol{u}}=\left[\begin{array}{c}
D G_{L M S} \cdot \Delta \boldsymbol{u}+D G_{L M S} \cdot \Delta \boldsymbol{w}+D G_{L M S} \cdot \Delta p_{w} \\
D G_{L M W} \cdot \Delta \boldsymbol{u}+D G_{L M W} \cdot \Delta \boldsymbol{w}+D G_{L M W} \cdot \Delta p_{w} \\
D G_{M B E} \cdot \Delta \boldsymbol{u}+D G_{M B E} \cdot \Delta \boldsymbol{w}+D G_{M B E} \cdot \Delta p_{w}
\end{array}\right],
$$

where $D G_{L M S}, D G_{L M W}$ and $D G_{M B E}$ represent the derivative of the linear momentum equations Eq. 15] and Eq. 16) of the solid phase and the fluid phase respectively and the derivative of the mass balance equation Eq. (17).

Rewritting Eq. (35) to separate the current $(k+1)$ and previous $(k)$ terms, since the later ones are not susceptible of linearization as they are constants that come from the previous step, we obtain:

$$
\begin{aligned}
\boldsymbol{G}_{k+1} & =\left[\alpha_{1} \boldsymbol{M}+\alpha_{4} \boldsymbol{C}\right] \hat{\boldsymbol{u}}_{k+1}+\boldsymbol{R}\left(\hat{\boldsymbol{u}}_{k+1}\right)-\boldsymbol{P}_{k+1} \\
& +\left[\alpha_{1} \boldsymbol{M} \hat{\boldsymbol{u}}_{k}-\alpha_{2} \boldsymbol{M} \dot{\hat{\boldsymbol{u}}}_{k}-\alpha_{3} \boldsymbol{M} \ddot{\hat{\boldsymbol{u}}}_{k}\right]+\left[\alpha_{4} \boldsymbol{C} \hat{\boldsymbol{u}}_{k}+\alpha_{5} \boldsymbol{C} \dot{\hat{\boldsymbol{u}}}_{k}+\alpha_{6} \boldsymbol{C} \ddot{\hat{\boldsymbol{u}}}_{k}\right]
\end{aligned}
$$


Consequently, after integration in time, Equations (15), (16) and (17) are written at time ${ }_{k+1}$ following Eq. (41) in order to be derived and linearized:

$$
\begin{gathered}
-\alpha_{1} \int_{\Omega} \delta \boldsymbol{u} \cdot\left[\rho \boldsymbol{u}+\rho_{w} \boldsymbol{w}\right] \mathrm{d} \Omega-\int_{\Omega} \boldsymbol{\sigma}^{\prime}: \operatorname{grad} \delta \boldsymbol{u} \mathrm{d} \Omega+\int_{\Omega} p_{w} \operatorname{div} \delta \boldsymbol{u} \mathrm{d} \Omega+\int_{\Omega} \delta \boldsymbol{u} \cdot \rho \boldsymbol{g} \mathrm{d} \Omega+\int_{\Gamma_{t}} \overline{\boldsymbol{t}} \cdot \delta \boldsymbol{u} \mathrm{d} \gamma=0 \\
-\int_{\Omega} \operatorname{grad}\left(p_{w}\right) \cdot \delta \boldsymbol{w} \mathrm{d} \Omega-\alpha_{4} \int_{\Omega} \boldsymbol{w} \cdot \mathbf{k}^{-1} \cdot \delta \boldsymbol{w} \mathrm{d} \Omega-\alpha_{1} \int_{\Omega} \rho_{w} \boldsymbol{u} \cdot \delta \boldsymbol{w} \mathrm{d} \Omega-\alpha_{1} \int_{\Omega} \rho_{w} \frac{\boldsymbol{w}}{n} \cdot \delta \boldsymbol{w} \mathrm{d} \Omega+\int_{\Omega} \rho_{w} \boldsymbol{g} \cdot \delta \boldsymbol{w} \mathrm{d} \Omega=0 \\
\alpha_{4} \int_{\Omega} \operatorname{div}(\boldsymbol{u}) \cdot \delta p_{w} \mathrm{~d} \Omega+\alpha_{4} \int_{\Omega} \operatorname{div}(\boldsymbol{w}) \cdot \delta p_{w} \mathrm{~d} \Omega+\alpha_{4} \int_{\Omega} \frac{p_{w}}{Q} \cdot \delta p_{w} \mathrm{~d} \Omega=0
\end{gathered}
$$

The results of the linearization process are given in Eqs. A23, A24 and A25] of the Appendix A, where the details are also described.

An interesting result that will be used later, is that, neglecting second order terms, the matrix of the linear system to be solved is:

where:

$$
\left[\begin{array}{ccc}
\mathbf{K}^{m a t}+\alpha_{1} \mathbf{M}^{s} & \alpha_{1} \mathbf{M}^{w} & \mathbf{Q} \\
\alpha_{1} \mathbf{M}^{w} & \alpha_{4} \boldsymbol{M}_{k}+\frac{\alpha_{1}}{n} \mathbf{M}^{w} & -\mathbf{R} \\
\alpha_{4} \mathbf{Q}^{T} & \alpha_{4} \mathbf{Q}^{T} & \frac{\alpha_{4}}{Q} \mathbf{M}_{p}
\end{array}\right]\left[\begin{array}{c}
\Delta \tilde{\mathbf{u}} \\
\Delta \tilde{\mathbf{w}} \\
\Delta \tilde{\mathbf{p}}_{w}
\end{array}\right]=-\boldsymbol{G}(\hat{\boldsymbol{u}})
$$

$$
\boldsymbol{K}^{m a t}=\int_{\Omega} \boldsymbol{B}^{T} \cdot \boldsymbol{c}^{e p} \cdot \boldsymbol{B} \mathrm{d} \Omega
$$

and $c^{e p}$ is the material constitutive matrix.

\section{3 | STABILIZATION OF THE DISCRETE SYSTEM OF EQUATIONS}

In this section the stabilization techniques employed to mitigate the problems caused by low order elements are detailed. The techniques carried out for both phases are treated separately; the stabilization of the mass balance equation, 3.1 is proposed for the improvement of the behavior of the water phase, meanwhile the solid pressure is stabilized through the volumetric deformation, 3.2

\section{1 | Stabilization of the mass balance equation}

To motivate the need for stabilization in the developed $\boldsymbol{u}-\boldsymbol{w}-p_{w}$ formulation, Equation 35, let us follow the reasoning exemplified by Zienkiewicz \& Taylor ${ }^{34}$. Assuming incompressible constituents, the Biot modulus $Q$ is large. Under that assumption the weak form of the mass balance equation given by Equation (19), (which is equivalent to Equation (17)) is considered, and Equation (45) reduced to:

$$
\left[\begin{array}{ccc}
\mathbf{K}^{m a t}+\alpha_{1} \mathbf{M}^{s} & \alpha_{1} \mathbf{M}^{w} & -\mathbf{Q} \\
\alpha_{1} \mathbf{M}^{w} & \alpha_{4} \boldsymbol{M}_{k}+\frac{\alpha_{1}}{n} \mathbf{M}^{w} & -\mathbf{R} \\
\alpha_{4} \mathbf{Q}^{T} & -\alpha_{4} \mathbf{R}^{T} & \mathbf{0}
\end{array}\right]\left[\begin{array}{c}
\Delta \tilde{\mathbf{u}} \\
\Delta \tilde{\mathbf{w}} \\
\Delta \tilde{\mathbf{p}}_{w}
\end{array}\right]=-\boldsymbol{G}(\hat{\boldsymbol{u}})
$$

which has the same formal structure than that of optimization problems in which constraints are imposed by the method of Lagrange multipliers. As such, in order to have a non-singular system and, consequently, a water pressure field free of spurious oscillations, the following conditions must be fulfilled:

$$
n_{u} \geq n_{p_{w}} \quad n_{w} \geq n_{p_{w}}
$$

being $n_{u}, n_{w}$ and $n_{p_{w}}$ the number of degrees of freedom for displacement, water displacement and water pressure fields. In addition to the usual condition required in the for the $\boldsymbol{u}-p_{w}$ problem, $n_{u} \geq n_{p_{w}}$ (see, for instance, Pastor et al ${ }^{[37}$ ), and additional condition arises from the Darcy's problem: $n_{w} \geq n_{p_{w}}$. These conditions, although necessary, are not sufficient and must be fulfilled in any 
assembly of elements of the mesh 34 37. A more rigorous study of the stability conditions of the three-field formulation may be found elsewhere 38 .

Using a $\boldsymbol{u}-p_{w}$ formulation, it is well known that the pore pressure field tend to exhibit oscillations, which tend to increase when the time step is reduced ${ }^{39}$. As shown in the one-dimensional (Terzhagi's equation) analysis of the discrete equations, the use of implicit time-integration scheme produce a stiffness matrix whose eigenvalues are positive and, consequently, the overall algorithm is unconditionally stable. However, the water pressure field may exhibit oscillations if the time step is smaller than:

$$
\Delta t \leq \frac{h^{2}}{6 c_{v}}
$$

where $c_{v}=M k$ is the coefficient of consolidation, $M$ is the constrained modulus and $h$ the element size. By numerical examples, it can be shown that this limit also applies to two- and three-dimensional analysis ${ }^{39440}$.

On the contrary, the structure of the system matrix, Equation (47), allows to hypothesize that the $\boldsymbol{u}-\boldsymbol{w}-p_{w}$ formulation may suffer spurious spatial oscillations of the water pressure field irrespectively of the oedometric modulus and the time-step. The reason beneath this fact is that the system matrix of the $\boldsymbol{u}-\boldsymbol{w}-p_{w}$ formulation always has the same form that the Lagrangian problem (irrespectively of the value of the permeability); differently, using the $\boldsymbol{u}-p_{w}$ formulation, the term $K_{p_{w} p_{w}}$ is significantly different from zero when the permeability or the time-step are large (i.e, when the time-step is larger than the critical time-step depicted in Equation (49) ) .

\subsection{1 | Polynomial pressure projection}

The Polynomial Pressure Projection (PPP) stabilization method has been originally developed to stabilize Stokes equations $s^{41116}$ and has also been applied to stabilize the $\mathbf{u}-p$ mixed formulation of Solid Mechanics ${ }^{42}$ and the simplified $\boldsymbol{u}-p_{w}$ formulation

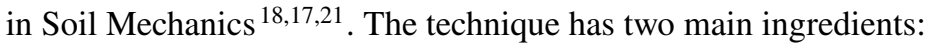

1. A mixed equal order interpolation of the scalar and vector fields.

2. A $L_{2}$ projection of the scalar variables.

Stabilization is achieved by adding to the mass balance equation a term proportional to the difference between the water pressure field and its projection to a polynomial of lower order. Unlike other stabilization methods, high-order derivatives are not required and it can be implemented at element level. Given a function $p \in L_{2}$, the $L_{2}$ projection operator $\breve{p}: L_{2} \rightarrow Q^{0}$ is defined by

$$
\int_{\Omega_{0}} \delta \breve{q}(p-\breve{p}) \mathrm{d} \Omega_{0}=0 \quad \forall \delta \breve{q} \in Q^{0}
$$

where $\breve{p}$ is the best approximation of the pressure $p$ in the space of polynomials of order $\mathcal{O}\left(Q^{0}\right)$.

Then, the stabilization term reads:

$$
\int_{\Omega_{0}^{e}}(\delta q-\delta \breve{q}) \tau(p-\breve{p}) \mathrm{d} \Omega_{0}=\int_{\Omega_{0}^{e}} \tau(\delta q p-\delta \breve{q} \breve{p}) \mathrm{d} \Omega_{0}
$$

where $\tau$ is the stabilization parameter. As shown in Appendix B this parameter may be estimated through:

$$
\tau= \begin{cases}\frac{2}{M}+\frac{2}{Q}-\frac{\beta}{\gamma} \frac{12 \Delta t k}{h^{2}} & \text { if } \Delta t \leq \frac{\gamma}{\beta} \frac{h^{2}}{6}\left(\frac{1}{c_{v}}+\frac{1}{k Q}\right) \\ 0 & \text { other cases }\end{cases}
$$

that is, it depends on the coefficient of consolidation, $c_{v}$, the element size, $h$, and the time integration method through the time step, $\Delta t$, and Newmark parameters, $\beta$ and $\gamma$.

\subsubsection{Stabilization with the Divergence of Momentum Equation (DME)}

In the context of Computational Fluid Dynamics, several stabilization techniques have been proposed. On the one hand, Brezzi and Pitkaranta ${ }^{43}$ proposed to add, in the $\boldsymbol{K}_{p p}$ position (the position equivalent to $\boldsymbol{K}_{p_{w} p_{w}}$ of the current formulation), the term:

$$
h \int_{\Omega}(\nabla N)^{T} \cdot \nabla N d \Omega
$$


Interestingly, this term tends to zero as the mesh is refined; thus, consistency is preserved. Later, Hughes et al ${ }^{11}$ introduced developed an stabilization technique based on Petrov-Galerkin; the key idea is adding a term proportional to the product of the linear momentum equation by $\frac{h^{2}}{2 \mu} \nabla N$ to the mass balance equation. On the other hand, Hafez and Soliman $\frac{15}{15}$ proposed to stabilize the Navier-Stokes equations by adding the divergence of the momentum equation to the mass balance equation. This method has been adapted to the hydromechanical problem in quasi-static conditions ( $\boldsymbol{u}-p_{w}$ formulation) by Pastor et al ${ }^{37}$. That work is here extended to a formulation valid for the full Biot equations.

In our case, the divergence of the time derivative of the linear momentum balance equation of the mixture, multiplied by the parameter $\alpha$, yields:

$$
\alpha\left[\nabla \cdot\left(\nabla \cdot \dot{\boldsymbol{\sigma}}^{\prime}\right)-\nabla \cdot\left(\nabla \dot{p}_{w}\right)-\rho \nabla \cdot \frac{\mathrm{d} \ddot{\boldsymbol{u}}}{\mathrm{d} t}-\rho_{w} \nabla \cdot \frac{\mathrm{d} \ddot{\boldsymbol{w}}}{\mathrm{d} t}\right]=0
$$

The divergence of the time derivative of the linear momentum balance equation of the fluid, necessary in subsequent derivations, can be written as:

$$
-\nabla \cdot\left(\nabla \dot{p}_{w}\right)-\frac{1}{k} \nabla \cdot \ddot{\boldsymbol{w}}-\rho_{w} \nabla \cdot \frac{\mathrm{d} \ddot{\boldsymbol{u}}}{\mathrm{d} t}-\frac{\rho_{w}}{n} \nabla \cdot \frac{\mathrm{d} \ddot{\boldsymbol{w}}}{\mathrm{d} t}=0
$$

The second time derivative of the mass balance equation allows us to get the last terms of Eqs. (54) and (55):

$$
\nabla \cdot \frac{\mathrm{d} \ddot{u}}{\mathrm{~d} t}+\nabla \cdot \frac{\mathrm{d} \ddot{\boldsymbol{w}}}{\mathrm{d} t}+\frac{1}{Q} \frac{\mathrm{d} \ddot{p}_{w}}{\mathrm{~d} t}=0
$$

Considering that the mixture compressibility, $Q$, is large, the last term can be neglected and the following relationship may be found:

yielding Eqs. (54) and (55):

$$
-\nabla \cdot \frac{\mathrm{d} \ddot{u}}{\mathrm{~d} t}=\nabla \cdot \frac{\mathrm{d} \ddot{w}}{\mathrm{~d} t}
$$

$$
\begin{gathered}
\alpha\left[\nabla \cdot\left(\nabla \cdot \dot{\boldsymbol{\sigma}}^{\prime}\right)-\nabla \cdot\left(\nabla \dot{p}_{w}\right)+\left(\rho-\rho_{w}\right) \nabla \cdot \frac{\mathrm{d} \ddot{\boldsymbol{w}}}{\mathrm{d} t}\right]=0 \\
\rho_{w} \frac{n-1}{n} \nabla \cdot \frac{\mathrm{d} \ddot{\boldsymbol{w}}}{\mathrm{d} t}=\nabla \cdot\left(\nabla \dot{p}_{w}\right)+\frac{1}{k} \nabla \cdot \ddot{\boldsymbol{w}}
\end{gathered}
$$

Thus, the divergence of time derivative of $\ddot{\boldsymbol{w}}$ in Eq. (58) can be substituted with the definition of the Eq. (59), allowing us to obtain the equation:

$$
\begin{array}{r}
\alpha\left[\nabla \cdot\left(\nabla \cdot \dot{\boldsymbol{\sigma}}^{\prime}\right)-\nabla \cdot\left(\nabla \dot{p}_{w}\right)+\frac{\rho-\rho_{w}}{\rho_{w}} \frac{n}{n-1}\left(\nabla \cdot\left(\nabla \dot{p}_{w}\right)+\frac{1}{k} \nabla \cdot \ddot{\boldsymbol{w}}\right)\right]= \\
\alpha\left[\nabla \cdot\left(\nabla \cdot \dot{\boldsymbol{\sigma}}^{\prime}\right)-\nabla \cdot\left(\nabla \dot{p}_{w}\right)\right]+\tau \nabla \cdot\left(\nabla \dot{p}_{w}\right)+\frac{\tau}{k} \nabla \cdot \ddot{\boldsymbol{w}}
\end{array}
$$

where $\tau=\alpha \frac{\rho-\rho_{w}}{\rho_{w}} \frac{n}{n-1}$.

The first term can be approximated by using different computational techniques (see ${ }^{44}$ ). However, it is interesting to note that the hypothesis of constant total stress over time, $\dot{\boldsymbol{\sigma}}=\mathbf{0}$, yields: $\nabla \cdot(\nabla \cdot \dot{\boldsymbol{\sigma}})=\nabla \cdot\left(\nabla \cdot \dot{\boldsymbol{\sigma}}^{\prime}-\nabla \dot{p}_{w}\right)=\mathbf{0}$. Finally, the stabilization term to add to the mass balance equation reads:

$$
\tau \nabla \cdot\left(\nabla \dot{p}_{w}\right)+\frac{\tau}{k} \nabla \cdot \ddot{\boldsymbol{w}}
$$

Pastor and coworkers proposed the following definition of the parameter $\alpha$ :

$$
\alpha=\tau_{0} \frac{\Delta t_{c r i t}^{2}}{\rho}
$$

where $\Delta t_{\text {crit }}^{2}$ is the critical time step employed in order to capture the $\mathrm{P}$ wave in the drained material taking into account the element size $h$ and the characteristic P-wave velocity, $V_{c}$. Rearranging (62) we obtain:

$$
\alpha=\tau_{0} \frac{h^{2}}{V_{c}^{2} \rho}=\tau_{0} \frac{h^{2}}{\frac{M}{\rho} \rho}=\tau_{0} \frac{h^{2}}{M} \quad \rightarrow \quad \tau=\tau_{0} \frac{h^{2}}{M} \frac{\rho-\rho_{w}}{\rho_{w}} \frac{n}{n-1}
$$

After applying the finite element discretization to the proposed stabilization technique, Equation 61, the semi-discrete equation to be added to the balance equations, Equation (31), reads:

$$
\tau \boldsymbol{K}_{p}^{s} \cdot \dot{\hat{\boldsymbol{p}}}_{w}+\frac{\tau}{k} \boldsymbol{Q}^{T} \cdot \ddot{\hat{\boldsymbol{w}}}
$$

where

$$
\boldsymbol{K}_{p}^{s}=\int_{\Omega}(\nabla \boldsymbol{N})^{T} \cdot \nabla \boldsymbol{N} d \Omega
$$




\section{2 | Stabilization of solid induced volumetric locking}

Stabilized mixed formulations have been proposed for hydromechanical problems to mitigate solid induced volumetric locking under quasi-static conditions ( $\mathbf{u}-p_{w}$ formulation); see, for instance, Sun et al ${ }^{1820}$ and Monforte et al ${ }^{21}$. In this work the socalled $\boldsymbol{u}-\theta-p_{w}$ mixed formulation presented by Monforte et al ${ }^{21}$ is extended to the dynamic range, resulting in a $\boldsymbol{u}-\theta-\boldsymbol{w}-p_{w}$ formulation. The gist is quite simple: first, a new variable $(\theta)$ is introduced to approximate volumetric deformation and then employed to define an assumed deformation gradient; second, the constitutive model is evaluated with this newly introduced assumed deformation gradient. Using this scheme the usual form of strain-driven stress integration schemes may be maintained without modification.

The strong form of the $\boldsymbol{u}-\theta-\boldsymbol{w}-p_{w}$ formulation reads:

$$
\left\{\begin{array}{l}
\operatorname{div}\left(\breve{\boldsymbol{\sigma}}^{\prime}-p_{w} \mathbf{I}\right)-\rho \ddot{\boldsymbol{u}}-\rho_{w} \ddot{\boldsymbol{w}}+\rho \mathbf{g}=\mathbf{0} \\
J-\theta=0 \\
-\operatorname{grad} p_{w}-\boldsymbol{k}^{-1} \cdot \dot{\boldsymbol{w}}+\rho_{w}\left(\boldsymbol{g}-\ddot{\boldsymbol{u}}-\frac{\ddot{\boldsymbol{w}}}{n}\right)=\mathbf{0} \\
\operatorname{div}(\dot{\boldsymbol{u}})+\operatorname{div}(\dot{\boldsymbol{w}})+\frac{1}{Q} \dot{p}_{w}=0
\end{array}\right.
$$

where $J=\operatorname{det}(\boldsymbol{F})$ is the determinant of the deformation gradient and $\breve{\boldsymbol{\sigma}}^{\prime}=\hat{\boldsymbol{\sigma}}^{\prime}(\breve{\boldsymbol{F}}, V)$ is the Cauchy stress tensor evaluated with the assumed deformation gradient, $\breve{\boldsymbol{F}}$. Initial and boundary conditions are analogous to those in Equations $(12)$ and not written for brevity.

The assumed deformation gradient, $\breve{\mathbf{F}}$, is defined as:

$$
\breve{\mathbf{F}}=\breve{\mathbf{F}}^{v} \cdot \mathbf{F}^{d}=\underbrace{\left(\theta^{\frac{1}{3}} \mathbb{1}\right)}_{\breve{\mathbf{F}}^{v}} \cdot \underbrace{\left(\operatorname{det}(\mathbf{F})^{-\frac{1}{3}} \mathbf{F}\right)}_{\mathbf{F}^{d}}=\left(\frac{\theta}{\operatorname{det}(\mathbf{F})}\right)^{\frac{1}{3}} \mathbf{F}
$$

That is, the deviatoric part of the deformation gradient, $\mathbf{F}^{d}$, is preserved whereas the volumetric part, $\breve{\mathbf{F}}^{v}$, is replaced with the $\theta$ variable. Note that in this formulation the Cauchy stress tensor depends on the displacements and on the Jacobian. Despite that, the usual form of strain-driven stress integration schemes is completely suitable for this formulation.

For plane strain conditions, the assumed deformation gradient, Equation (67), has to be redefined to guarantee that the out of plane component of the deformation is equal to unity. As in the $\overline{\mathbf{F}}$-method (F-bar method), the two-dimensional assumed deformation gradient is then $45 \mid 46$ :

$$
\breve{\mathbf{F}}^{2 D}=\left(\frac{\theta}{\operatorname{det}(\mathbf{F})}\right)^{\frac{1}{2}} \mathbf{F}^{2 D}
$$

And the constitutive model is evaluated with the three dimensional assumed deformation gradient:

$$
\breve{\mathbf{F}}^{C L}=\left(\begin{array}{cc}
\breve{\mathbf{F}}^{2 D} & \overrightarrow{0}^{T} \\
\overrightarrow{0} & 1
\end{array}\right)
$$

The use of a mixed formulation is not enough by itself to mitigate the high amplitude oscillations in the effective mean stress field if equal order interpolants are used for all the variables. Therefore a stabilization technique is also required here. For simplicity, PPP is also used, thus adding a stabilization term to the volume deformation balance equation. As such, the final form of the semi-discrete finite element equations of the $\boldsymbol{u}-\theta-\boldsymbol{w}-p_{w}$ reads:

$$
\left[\begin{array}{cccc}
\mathbf{M}^{s} & \mathbf{0} & \mathbf{M}^{w} & \mathbf{0} \\
\mathbf{0} & \mathbf{0} & \mathbf{0} & \mathbf{0} \\
\mathbf{M}^{w} & \mathbf{0} & \frac{1}{n} \mathbf{M}^{w} & \mathbf{0} \\
\mathbf{0} & \mathbf{0} & \mathbf{0} & \mathbf{0}
\end{array}\right]\left[\begin{array}{c}
\ddot{\tilde{\mathbf{u}}} \\
\ddot{\tilde{\boldsymbol{\theta}}} \\
\ddot{\tilde{\mathbf{w}}} \\
\tilde{\mathbf{p}}_{w}
\end{array}\right]+\left[\begin{array}{cccc}
\mathbf{0} & \mathbf{0} & \mathbf{0} & \mathbf{0} \\
\mathbf{0} & \mathbf{0} & \mathbf{0} & \mathbf{0} \\
\mathbf{0} & \mathbf{0} & \mathbf{M}_{k} & \mathbf{0} \\
\mathbf{Q}^{T} & \mathbf{0} & \mathbf{Q}^{T} & \frac{1}{Q} \mathbf{M}_{p}
\end{array}\right]\left[\begin{array}{c}
\dot{\tilde{\mathbf{u}}} \\
\dot{\tilde{\boldsymbol{\theta}}} \\
\dot{\tilde{\mathbf{w}}} \\
\tilde{\mathbf{\mathbf { p }}}_{w}
\end{array}\right]=\left[\begin{array}{c}
\mathbf{f}_{s}^{e x t}-\mathbf{f}^{i n t}\left(\breve{\boldsymbol{\sigma}}^{\prime}\right)+\mathbf{Q} \cdot \tilde{\mathbf{p}}_{w} \\
\mathbf{f}^{\theta}-\left(\mathbf{M}_{\theta}+\alpha_{\theta} \mathbf{M}_{\theta}^{s}\right) \cdot \tilde{\boldsymbol{\theta}} \\
\mathbf{f}_{w}^{e x t}-\mathbf{R} \cdot \tilde{\mathbf{p}}_{w} \\
\mathbf{0}
\end{array}\right]
$$

where the stabilization factor for the volume deformation balance equation is $\alpha_{\theta}$ and:

$$
\mathbf{f}^{\theta}=\int_{\Omega_{t}} \mathbf{N}^{T} \mathrm{~d} \Omega_{t}
$$




$$
\begin{gathered}
\mathbf{M}_{\theta}=\int_{\Omega} \mathbf{N}^{T} \cdot \mathbf{N} \frac{1}{J} \mathrm{~d} \Omega \\
\mathbf{M}_{\theta}^{s}=\int_{\Omega} \mathbf{N}^{T} \cdot \mathbf{N} \frac{1}{J} \mathrm{~d} \Omega-\int_{\Omega} \breve{\mathbf{N}}^{T} \cdot \breve{\mathbf{N}} \frac{1}{J} \mathrm{~d} \Omega
\end{gathered}
$$

where $\breve{\mathbf{N}}$ are the set of polynomials introduced in Equation (51); in the case of linear triangles, these local element polynomials are $\breve{\mathbf{N}}^{e}=[1 / 3,1 / 3,1 / 3]$.

Time integration of the stabilized balance equations, Equation (70) follows the scheme describe above (section 2.2.2) for the $\boldsymbol{u}-\boldsymbol{w}-p_{w}$ formulation.

\section{4 | REPRESENTATIVE NUMERICAL SIMULATIONS}

The methodology described in the previous sections has been incorporated into G-PFEM, a code developing geotechnical applications of PFEM (Monforte et al. ${ }^{14 / 21 / 29}$ ). G-PFEM is implemented into Kratos (Dadvand et al.$^{47}$ ), an object-oriented multidisciplinary open-access platform for development of numerical analysis tools. Several numerical examples were run to test the capabilities of the newly implemented algorithms, with a particular focus on the performance of the stabilization techniques. The numerical test cases were selected to allow validation against analytical solutions or, at least, comparison with previously published results of similar codes.

The examples explore a large range of coupled dynamic problems. This can be visualized (Figure 1 ) in a plane spanned by the dimensionless variables $\Pi_{1}$ and $\Pi_{2}$, defined as:

$$
\Pi_{1}=k \frac{M+Q}{\omega H_{T}^{2}}, \quad \Pi_{2}=\frac{\omega^{2} H_{T}^{2} \rho}{M+Q}
$$

where $\omega$ is the angular velocity of the load, $H_{T}$ is the column height and $M$ and $Q$ represent the constrained modulus of the soil and the bulk modulus of the mixture respectively. For given problem dimensions $\Pi_{1}$ and $\Pi_{2}$ are mostly controlled by the permeability of the soil and the loading frequency, respectively.

These dimensionless variables were introduced by Zienkiewicz and coworkers 48 to analyze a problem of one-dimensional dynamic consolidation under harmonic loading. As a result zones in the plane $\Pi_{1}$ and $\Pi_{2}$ were drawn to indicate the range of application of different simplifications of Biot equations (Figure 1 . Zone I would correspond to quasi-static conditions, in which inertial terms made little difference. Zone II to conditions in which relative fluid-solid acceleration could be neglected and the $\boldsymbol{u}-p_{w}$ formulation was appropriate. In zone III a full Biot formulation was necessary. The analysts also indicated that $\Pi_{1}$ values above $1 \mathrm{e} 2$ were indicative of drained conditions and below 1e-2 of undrained conditions. Recently Navas et al. .4950 using a meshfree method at large strain have observed that this zoning scheme is also valid for two-dimensional problems.

\section{1 | Soil column: ramped-loading case}

The first example corresponds to semi-instantaneous (ramped) loading at the upper boundary of a soil column. This, of course, is the loading condition for the incremental-loading oedometer test, which in the classical analysis by Terzaghi is solved neglecting inertial terms. This provides an analytical solution, useful as a reference. However, differences are to be expected, as the proposed methodology should capture the shock dynamics associated with this kind of loading, which are ignored in the classical analysis.

Indeed, a well-known consequence of Biot theory ${ }^{1151}$ is the apparition of two separate longitudinal modes of wave propagation. For the first one, the fastest, porous skeleton and the interstitial fluid move in phase, so that it can be captured by simplified formulations, in which relative acceleration of fluid phase is ignored. The opposite is true for the second wave, known also as Biot slow wave, in which solid and interstitial fluid move essentially out of phase. Verruijt ${ }^{52}$ gives the following expressions for the wave velocities of the two longitudinal modes:

$$
c_{1}^{2}=\frac{M+Q}{\rho}, \quad c_{2}^{2}=\left[\frac{n \frac{M}{Q}}{1-n+\frac{M}{Q}}\right] \frac{Q}{\rho_{w}}
$$

Two cases are presented, with increasing speed of load application. 


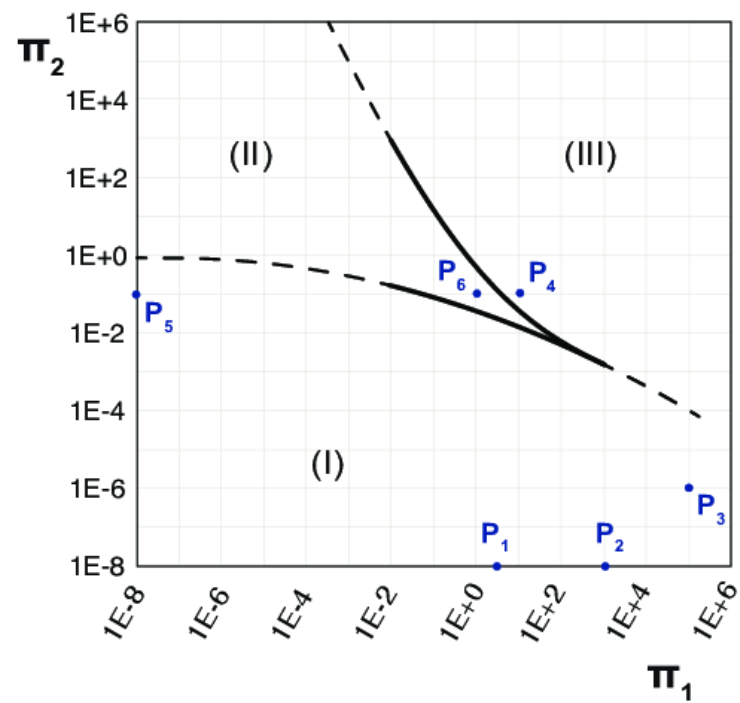

Zone (I) - Slow phenomena: ü and w can be neglected

Zone (II) - Moderate speed: $\ddot{\text { w }}$ can be neglected

Zone (III) - Fast phenomena: only full Biot eq. valid

FIGURE 1 Location of each numerical example within the Zienkiewickz et al. ${ }^{48}$ dimensionless plane.

TABLE 1 Material parameters of the ramped-loading consolidation problem (Sections 4.1.1 and 4.1.2)

\begin{tabular}{c|cccccccc}
\hline Section & $E[\mathrm{MPa}]$ & $v$ & $K_{w}[\mathrm{MPa}]$ & $K_{s}[\mathrm{MPa}]$ & $n$ & $\kappa[\mathrm{m} / \mathrm{s}]$ & $\rho_{w}\left[\mathrm{~kg} / \mathrm{m}^{3}\right]$ & $\rho_{s}\left[\mathrm{~kg} / \mathrm{m}^{3}\right]$ \\
\hline 4.1 .1 & 4.0 & 0.2857 & $10^{4}$ & $10^{34}$ & 0.363 & $10^{-7}$ & 1000 & 2700 \\
\hline 4.1 .2 & $5.0 \cdot 10^{3}$ & 0.0 & $2.0 \cdot 10^{3}$ & $10^{34}$ & 0.4 & $10^{-3}$ & 1000 & 2650 \\
\hline
\end{tabular}

\subsection{1 | Slower loading rate}

The domain consists of an elastic material of 30 meters height, whose lower boundary is rigidly fixed and impermeable and upper boundary is fully drained and free to move. On the upper boundary of the domain a ramped load is applied, increasing till $t_{0}=0.1 \mathrm{~s}$ and then remaining constant. The finite element mesh consists of 162 linear triangle elements and 103 nodes divided in a regular grid of 20x2 elements. The key material properties are listed in 1 whereas an sketch of the problem is presented in Figure 2 a) and (b).

The oedometer problem has been frequently employed ${ }^{917737}$ to assess the accuracy of numerical consolidation models using the reduced $\boldsymbol{u}-p_{w}$ formulation. Unstable elements show large amplitude oscillations in the water pressure profile during the first steps. This is also observed here. Figure 3 depicts the vertical profiles of normalized water pressure, $p_{w} / p_{\max }$, at $\mathrm{t}=0.1 \mathrm{~s}$. Figure 3 (a) compares the results obtained with direct integration (i.e. without any stabilization technique) with those obtained using the PPP and DME methodologies. Direct integration results in the typical oscillatory profile of the pore pressure. Both stabilization techniques are able to eliminate the oscillations and give almost coincident results.

Dynamic effects are present in this application, as shown clearly in Figure 3 (b). The reference quasi-static Terzaghi solution, with pore pressure equal throughout the domain to the overburden stress, (except for a thin zone near the draining boundary) is only recovered when all the accelerations are forced to be zero. The observed differences in the normalized water pressure, $p_{w} / p_{\max }$ profile may be best understood considering wave propagation.

The wave speed of the slow Biot wave $c_{2}$ for this problem is in the order of $55 \mathrm{~m} / \mathrm{s}$. At $0.1 \mathrm{~s}$, when Figure 3 is plotted, this wave has traveled $5.5 \mathrm{~m}$, i.e. the initial disturbance front would be located at a normalized height of 0.82 , which is coincident with the peak overpressure of the figure. This overpressure peak cannot be captured if the relative acceleration between solid and fluid is ignored. The slight overpressure at the bottom of the sample is instead attributed to dynamic undrained effects and is captured even when relative solid-fluid acceleration is zero. 


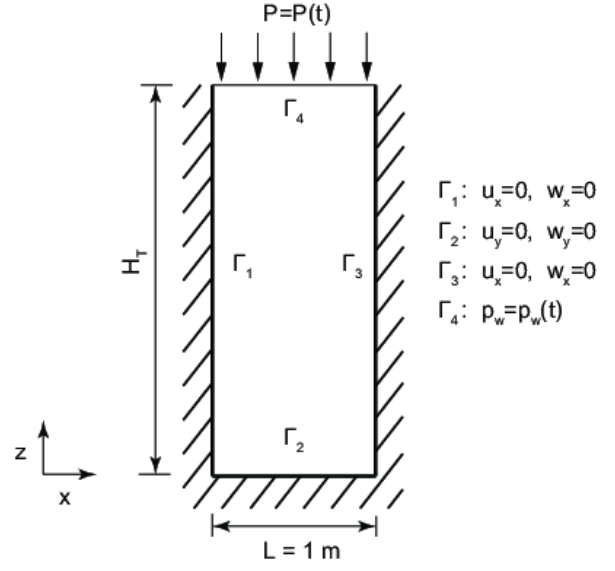

(a)

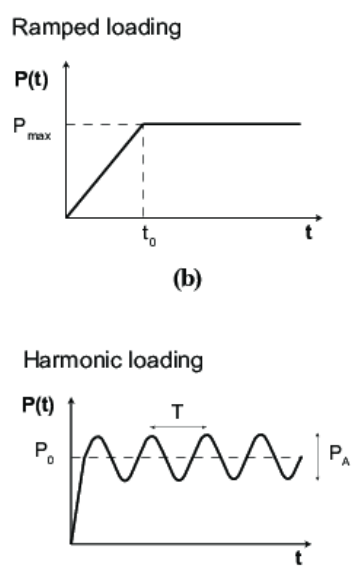

(c)

FIGURE 2 Oedometer scheme: (a) Geometry and boundary conditions; (b) Ramped-loading consolidation loading; and (c) Dynamic consolidation loading.

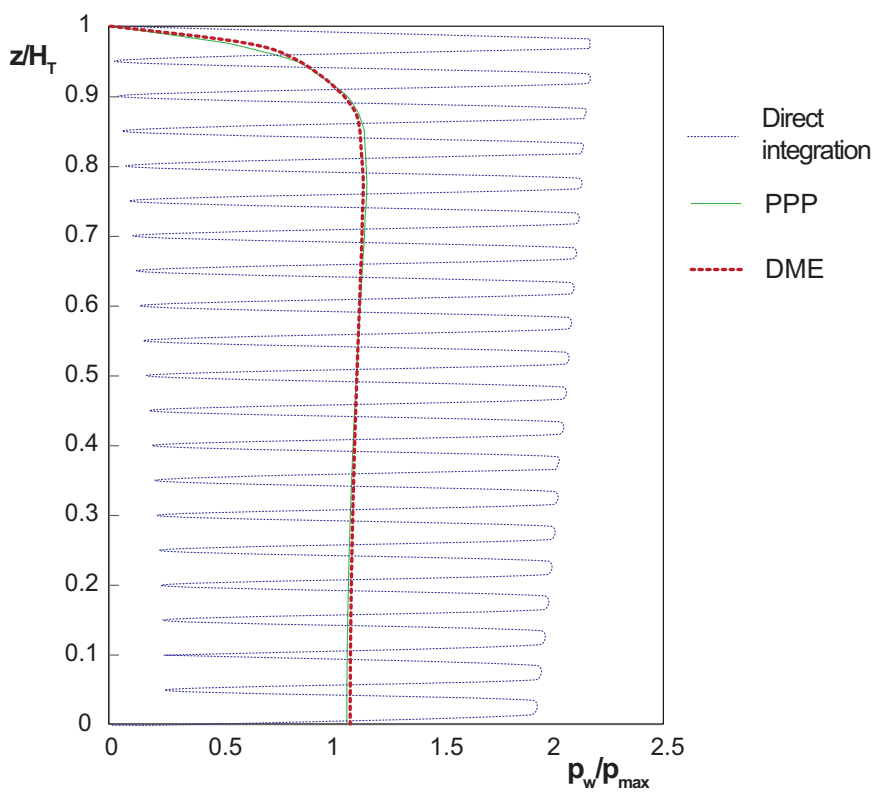

(a)

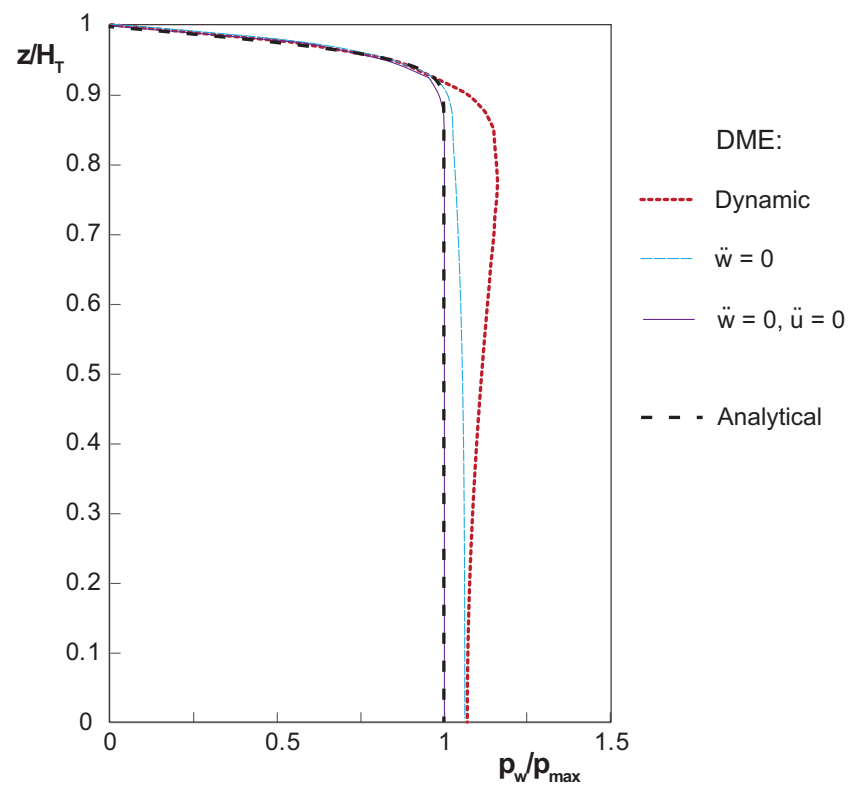

(b)

FIGURE 3 Ramped-loading with t0 $=0.1 \mathrm{~s}$ consolidation: (a) Comparison of stabilized and non-stabilized results; (b) Comparison between dynamic and non-dynamic formulations.

\subsection{2 | Faster loading rate}

In the case considered in this section the soil column is $10 \mathrm{~m}$ long and the loading step at the upper boundary is applied over a very small time period $\left(t_{0}=10 \mu \mathrm{s}\right)$. This case was also discussed by Sabetamal et al. ${ }^{24}$ following the analytical solutions proposed by Verruijt ${ }^{52}$ and Carter et al. .53 . The material parameters are listed in Table 1

Figure 4 represents the pore water pressure evolution at a depth of $0.2 \mathrm{~m}$ as obtained with the proposed solution in comparison with the analytical solution proposed by Verruijt ${ }^{52}$ and Carter et al. ${ }^{[3]}$ and the numerical one by Sabetamal et al. ${ }^{24}$. The time evolution clearly shows the arrival of the two compressive waves. It is clearly observed that the fast dilatational wave arrives at a time of approximately $90 \mu \mathrm{s}$, whereas the slower wave arrives after a delay of around $85 \mu \mathrm{s}$, having about one half of the 


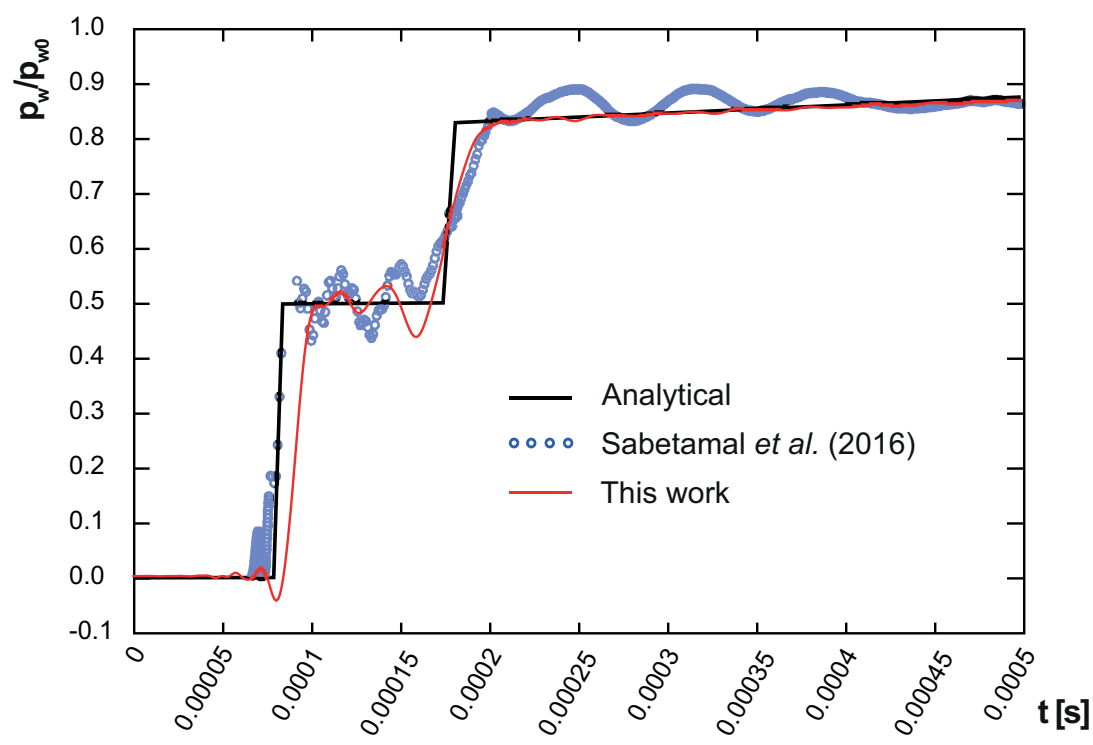

FIGURE 4 The evolution of pore water pressure at depth $0.2 \mathrm{~m}$ versus time.

TABLE 2 Material parameters of the cyclic-loading consolidation problem (Sections 4.2.1, 4.2.2 and 4.2.3)

\begin{tabular}{c|cccccccc}
\hline Section & $E[\mathrm{MPa}]$ & $v$ & $K_{w}[\mathrm{MPa}]$ & $K_{s}[\mathrm{MPa}]$ & $n$ & $k[\mathrm{~m} / \mathrm{s}]$ & $\rho_{w}\left[\mathrm{~kg} / \mathrm{m}^{3}\right]$ & $\rho_{s}\left[\mathrm{~kg} / \mathrm{m}^{3}\right]$ \\
\hline 4.2 .1 & 25.0 & 0.2 & $3.0 \cdot 10^{8}$ & $10^{34}$ & 0.3 & $10^{-7}, 10^{-5}$ & 1000 & 2000 \\
\hline 4.2 .2 & 30.0 & 0.2 & $3.3 \cdot 10^{8}$ & $10^{34}$ & 0.33 & $10^{-2}$ & 1000 & 2000 \\
\hline 4.2 .3 & 30.0 & 0.2 & $3.3 \cdot 10^{4}$ & $10^{34}$ & 0.33 & $1.5 \cdot 10^{-2}$ & 1000 & 2000 \\
\hline
\end{tabular}

velocity of the first wave. The solution obtained compares favorably with that presented by Sabetamal et al. ${ }^{24}$ and, despite some oscillations, shows good agreement with the analytical solution. Further improvement might be achieved exploring different numerical time integration schemes, but this is out of the scope of the present work.

\section{2 | Soil column: harmonic-loading case}

The second set of numerical simulations corresponds to a soil column under harmonic loading. Horizontal solid displacements as well as normal fluid motion are restrained on the lateral boundaries (see Figure 2 The loading curve shape is depicted in Figure 2 (c) and the material properties of the soil are listed in Table 2 The case is solved for low, intermediate and high frequency loading cases, as detailed below.

\subsubsection{Low frequency loading}

A reference study for this case was presented by De Pouplana and Oñate ${ }^{9}$ using a FIC based solution for the $\boldsymbol{u}-p_{w}$ formulation. Column height is $30 \mathrm{~m}$. The harmonic loading follows the equation: $q=1000+500 \sin (\omega t)[\mathrm{Pa}]$ with angular velocity, $\omega$, of 2 $\mathrm{rad} / \mathrm{s}$. Two different permeabilities are studied (Table 2 . These cases lie in zone I in Figure 1 so in principle inertial terms are not relevant.

Figure 5 presents pore pressure history at 1.5 meters below the top. Both stabilization techniques (PPP and DME) show an excellent agreement with the reference solution ${ }^{9}$. In the case with lowest permeability,(see Figure 5 (a)) pore pressure follows closely the applied load and conditions are practically undrained. If stabilization is not applied spurious values of excess water pressure are obtained (with peak values close to $2500 \mathrm{~Pa}$ ). For the case with larger permeability $\left(k=10^{-5} \mathrm{~m} / \mathrm{s}\right.$ ), the response in 


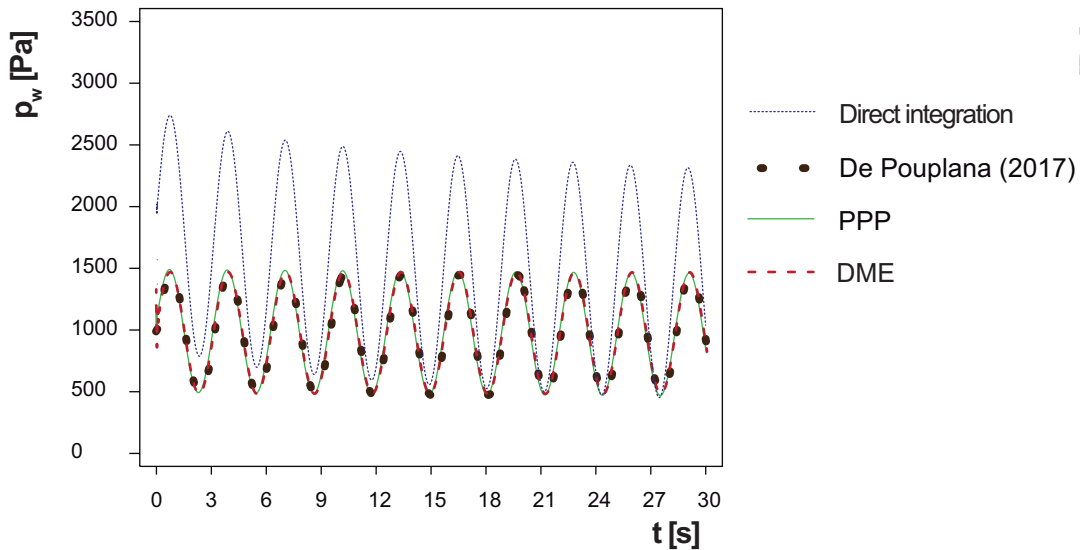

(a)

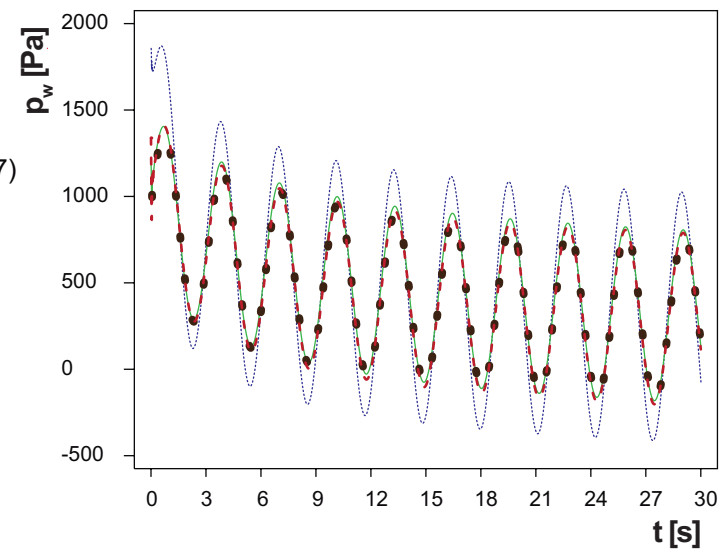

(b)

FIGURE 5 Low frequency harmonic-loading consolidation: Time evolution of pore pressure at location $(0.5,28.5)$ for (a) Undrained case $\left(\mathrm{k}=10^{-11} \mathrm{~m}^{2} / \mathrm{Pa} \cdot \mathrm{s}\right)$, (b) Semi-drained case $\left(\mathrm{k}=10^{-8} \mathrm{~m}^{2} / \mathrm{Pa} \cdot \mathrm{s}\right)$.

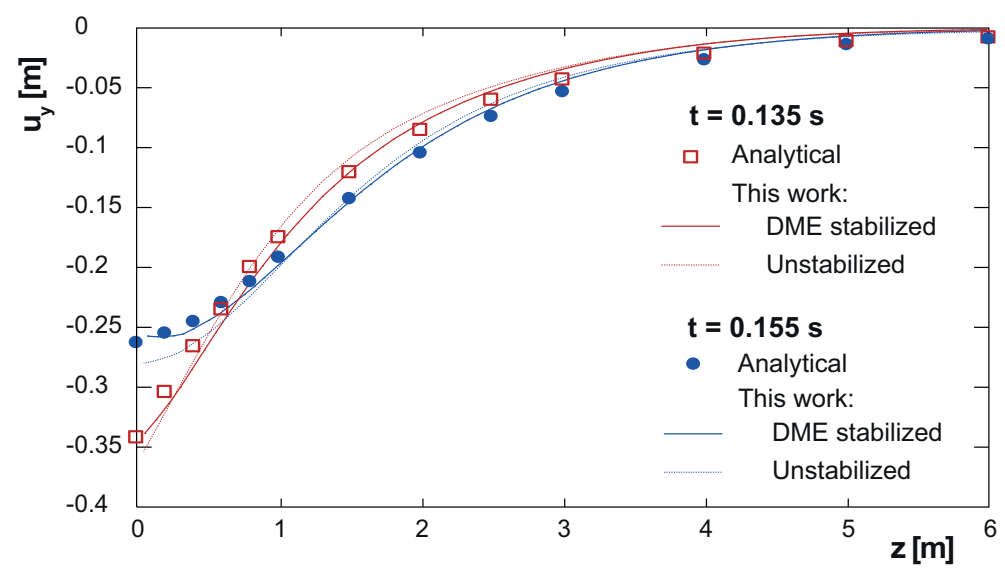

FIGURE 6 Intermediate frequency harmonic-loading consolidation: Solid displacement response versus depth.

partly drained and although the unstabilized formulation still introduces a significant error, its magnitude is relatively smaller (see Figure 5 (b)).

\subsection{2 | Intermediate frequency loading}

Sabetamal et al. ${ }^{[24}$ presented a case subject to intermediate frequency loading, given by $f(t)=3[1-\sin (\pi / 2+25 \pi t)] \mathrm{kPa}$. It was compared with the analytical solution proposed by De Boer ${ }^{54}$, corresponding to incompressible constituents. Column height is here $10 \mathrm{~m}$. The material properties, Table 2 correspond to an almost free draining material. Two snapshots of the displacement response as a function of depth are depicted in Figure 6 at two different times for both numerical and analytical solutions. They show good agreement between them and illustrate the robustness of the proposed methodology. As expected, the benefits of stabilization are less conspicuous in this drained case.

\subsection{3 । High frequency loading}

In this case the harmonic loading is as in the low frequency case but now angular velocity is $500 \mathrm{rad} / \mathrm{s}$. Column height is $5 \mathrm{~m}$. Permeability is relatively high at $1.5 \cdot 10^{-2} \mathrm{~m} / \mathrm{s}$ and fluid is made compressible to obtain a $\mathrm{Q}=10^{5} \mathrm{MPa}$. As indicated in Table 2 


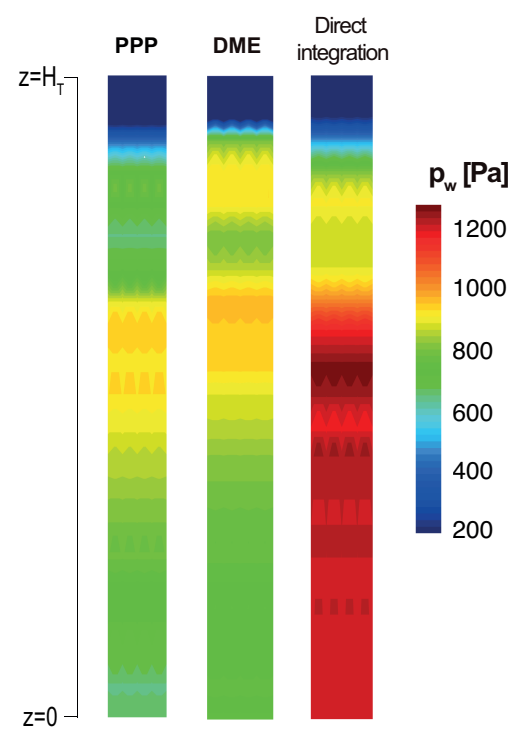

(a)

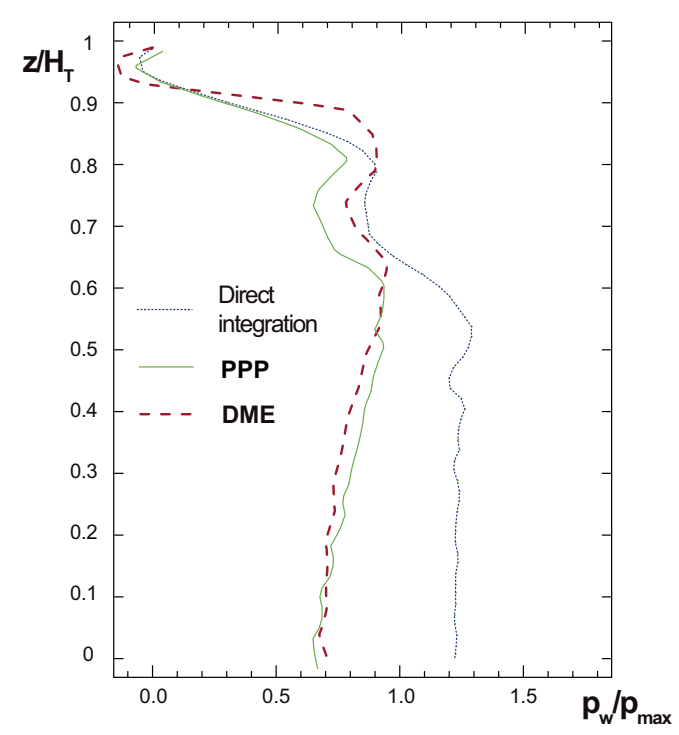

(b)

FIGURE 7 Performance of different numerical methodologies for high frequency loading: (a) pore pressure distribution at time $\mathrm{t}=0.020 \mathrm{~s}$., and (b) isochrone of normalized pore pressure at time $\mathrm{t}=0.020 \mathrm{~s}$.

this locates the case in Zone III (point 4 in Figure $1, \Pi_{1}=10, \Pi_{2}=10^{-1}$ ). There are no reference solutions that may be used for comparison in this case.

The pore pressure profile at $\mathrm{t}=0.02 \mathrm{~s}$ is illustrated in Figure 7 for computations using the two different stabilization techniques (PPP and DME) as well as the unstabilized algorithm. The unstabilized case clearly shows spurious high values of pore pressure, above the reference maximum pressure exerted at the upper boundary (see Figure 7 (b)) .

In the stabilized solutions two peaks of pore pressure appear in the normalized pore pressure graph (Figure $7 \mathrm{~b}$ )) . These are located roughly at 0.85 and 0.55 of column height. Considering that the slow wave speed $c_{2}$ is here $128 \mathrm{~m} / \mathrm{s}$ these two peaks are approximately one wavelength apart $(1.6 \mathrm{~m}$ at $\mathrm{f}=80 \mathrm{~Hz})$, as expected. Comparing the PPP and DME techniques it can be observed that PPP stabilization results in slightly higher dampening of the solution, whereas DME show somewhat higher diffusion. The dependency of these effects on aspects such as the stabilization parameter value are beyond the scope of the present work.

\section{3 | 2D wave propagation}

The last example examines 2-D wave propagation under plane-strain conditions, following closely a case discussed first by Breuer ${ }^{[5]}$ and later by Markert et al ${ }^{[23}$. The geometry, boundary conditions, loading history and soil parameters are presented in Fig. 8 A rectangular domain is loaded on its upper boundary by a harmonic half-cycle pressure pulse between 0 and $0.04 \mathrm{~s}$. The simulation is run for $0.2 \mathrm{~s}$ to minimize the effect of boundary reflections; these reflections might also be dealt by employing

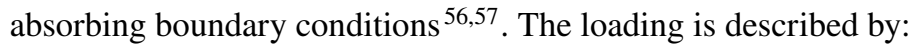

$$
F=10^{5} \sin (25 \pi t)[1-H(t-0.04)] \quad[\mathrm{Pa}],
$$

where $H(t-0.04)$ is the Heaviside function and $t$ is the current time in seconds. A mesh composed by 3960 nodes and 7670 low order triangular elements was employed. The load is applied at the 5 nodes closer to the symmetry axis.

Two cases with different permeabilities are studied, roughly corresponding to practically undrained and partially drained conditions, (points 5 and 6 in Figure 1 . For this second case the problem conditions lie in Zone II of Figure 1 As in previous works 55 [23, particular attention is paid at the evolution in points A and B (see Figure 8 of displacement and water pressure, respectively. 

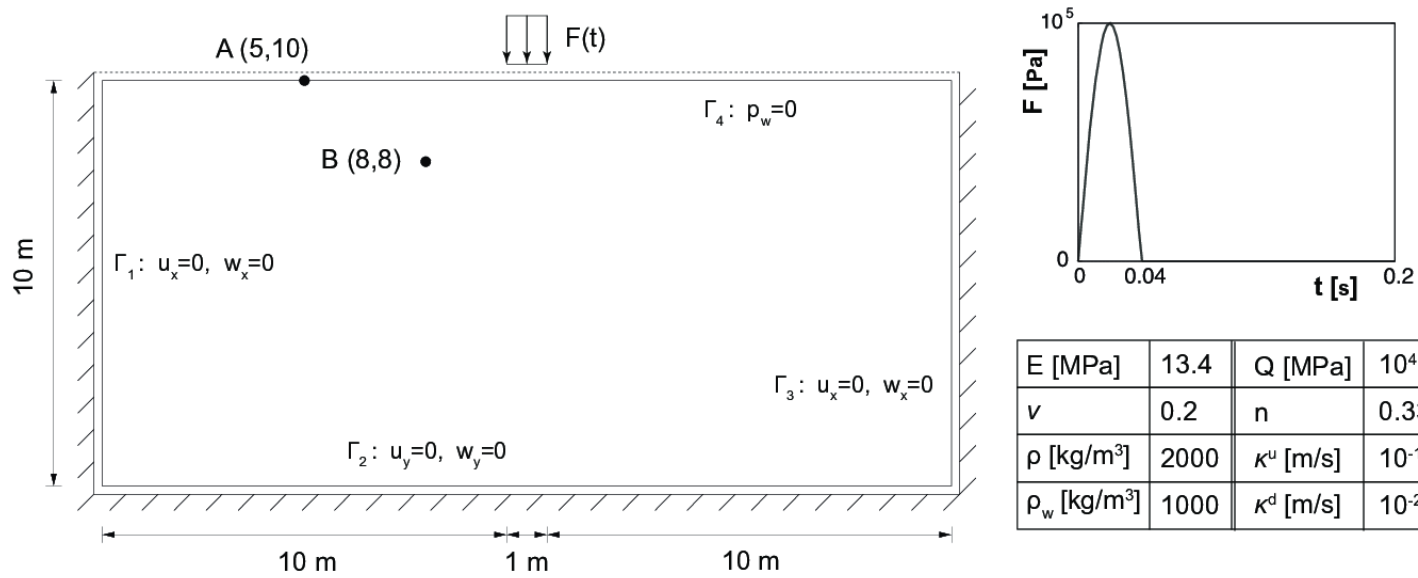

\begin{tabular}{|l|l||l|l|}
\hline$E[\mathrm{MPa}]$ & 13.4 & $Q[\mathrm{MPa}]$ & $10^{4}$ \\
\hline$v$ & 0.2 & $\mathrm{n}$ & 0.33 \\
\hline$\rho\left[\mathrm{kg} / \mathrm{m}^{3}\right]$ & 2000 & $K^{\mathrm{u}}[\mathrm{m} / \mathrm{s}]$ & $10^{-10}$ \\
\hline$\rho_{\mathrm{w}}\left[\mathrm{kg} / \mathrm{m}^{3}\right]$ & 1000 & $K^{\mathrm{d}}[\mathrm{m} / \mathrm{s}]$ & $10^{-2}$ \\
\hline
\end{tabular}

FIGURE 8 Geometry, boundary conditions, loading history and soil parameters of the 2D wave propagation case.

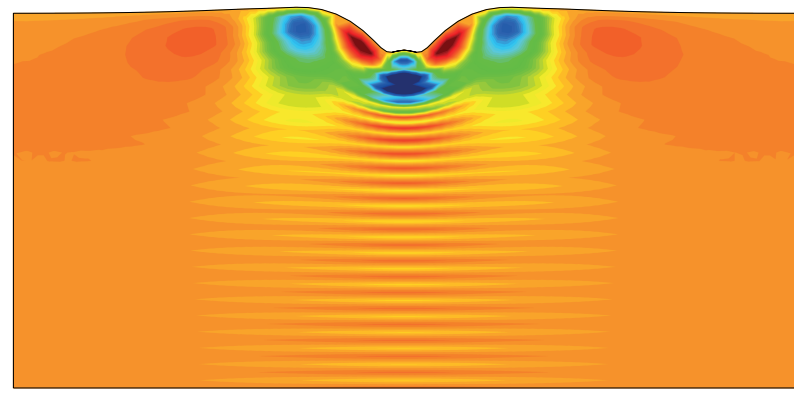

(a)

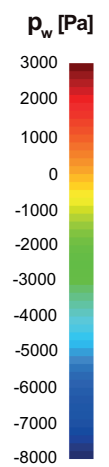

$-8000$

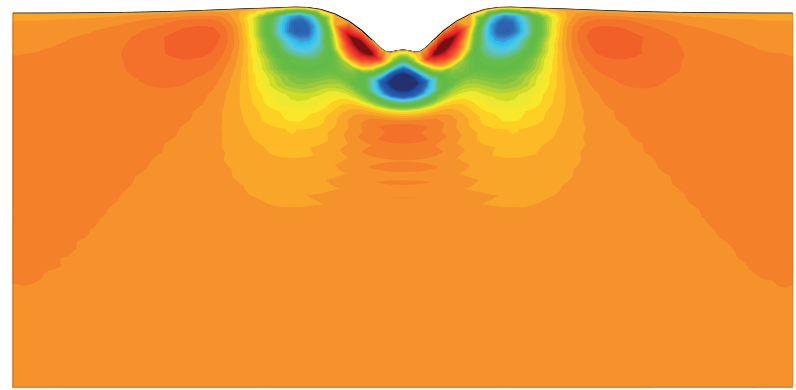

(b)

FIGURE $92 \mathrm{D}$ wave propagation in partially drained conditions. Solution at $t=0.05 \mathrm{~s}$. Without stabilization of the mass balance equation, (a), and with stabilization of the mass balance equation, (b).(Contour displacements magnified x 250).

Significant pore pressures are generated even in the partially drained case, Figure 9 although conditions are far from incompressibility. Even if the beneficial effect of stabilization (using DME with $\tau_{0}=0.1$ ) is clear in the spatial pore pressure pattern below the load, spurious water pressure oscillations still plays here a relatively minor role. This is visible in the comparative performance of different stabilization techniques, illustrated in Figure 10 The PPP solution was computed with $\Delta t=0.0025 \mathrm{~s}$., a value fixed by the condition given in Eq. $(\mathrm{B} 42)$. For the DME solution, several time steps were tried.

In Figure 10 a) the vertical displacement of point $\mathrm{A}$ is plotted against the horizontal displacement. The motion at $\mathrm{A}$ traces the classical quasi-elliptical retrogressive motion associated with Rayleigh wave propagation. There are some small differences in amplitude that reflect different amounts of numerical damping introduced by the different algorithms; only for the largest time step in DME the amount of damping is such to make a significant difference. Again, for this partially drained condition, the passage of the pore pressure wave at point B Fig. 10 b) appears only slightly affected by introducing stabilization or (for DME) by the time step. Only the PPP simulation shows some high frequency noise, near the wave peaks. Numerical dispersion (and subsequent wave widening) is somewhat larger in the formulations employed here than in the reference solution by Markert et $\mathrm{a}^{23}$. This is likely due to the different time integration algorithms employed.(The results illustrated from ${ }^{[23}$ were obtained with an implicit monolithic solution with quadratic approximation for the solid displacement and linear for pore pressure and water displacement and a composite 2nd order trapezoidal-backward difference time integration scheme). 


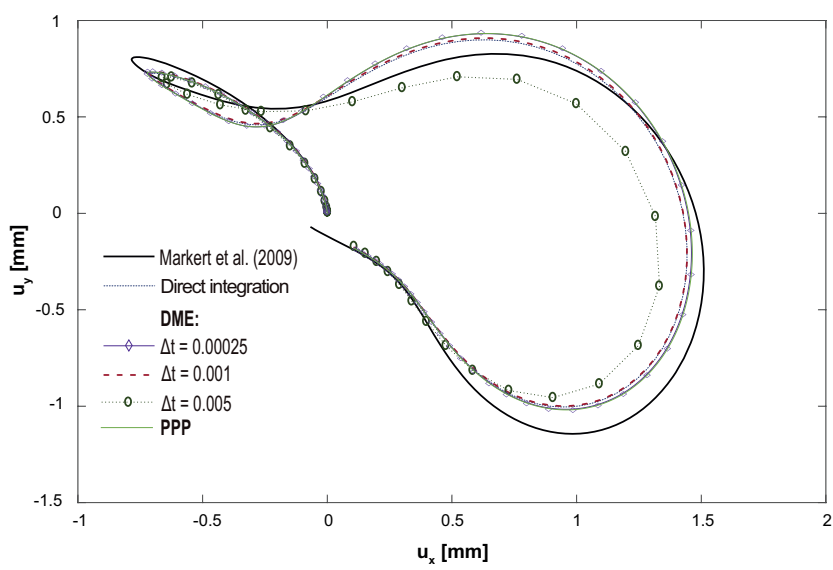

(a)

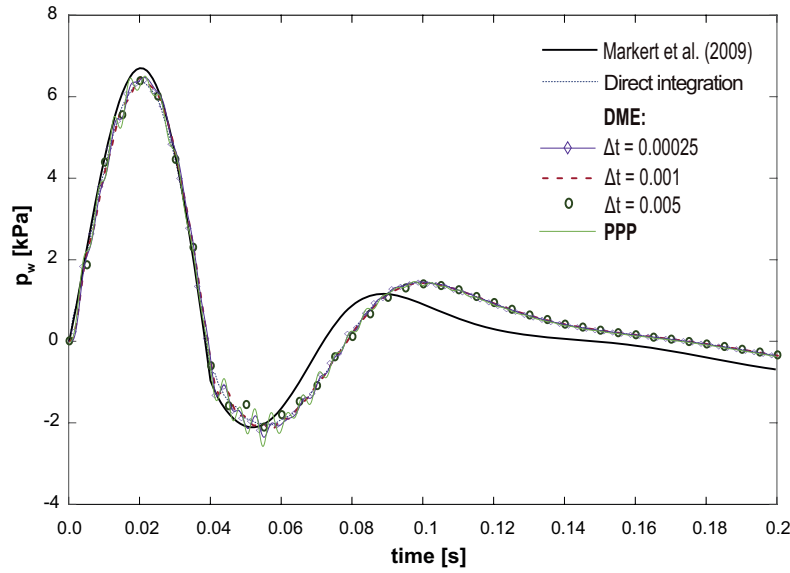

(b)

FIGURE 10 2D wave propagation in partially drained conditions: (a) In-plane motion at point $A$, and (b) pore pressure evolution at point $\mathrm{B}$.

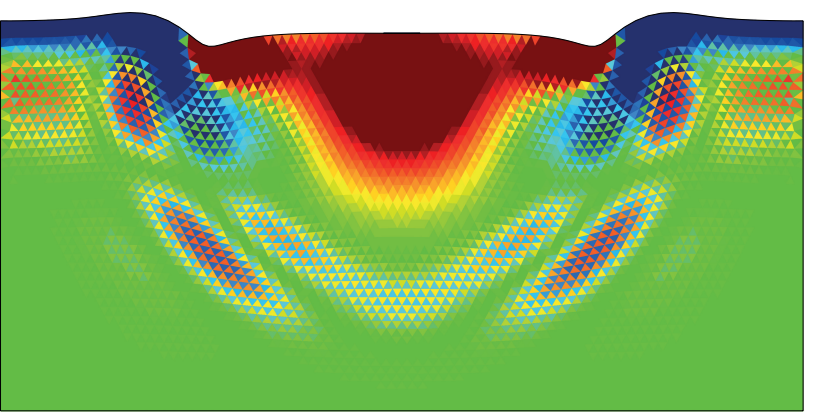

(a)

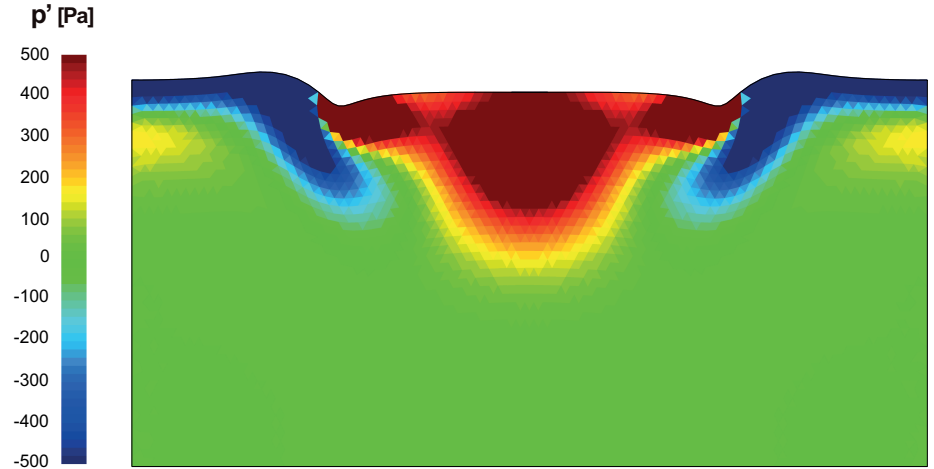

(b)

FIGURE $112 \mathrm{D}$ wave propagation in partially drained conditions Effective mean stress at $t=0.15 \mathrm{~s} . \boldsymbol{u}-\boldsymbol{w}-p_{w}$ formulation, (a), and $\boldsymbol{u}-\theta-\boldsymbol{w}-p_{w}$ formulation, (b). (Contour displacements magnified x 500).

The use of a stabilized mixed formulation to mitigate the solid-phase induced volumetric locking has significant effects in this partially drained case. The stabilization of the solid-phase induced volumetric locking has significant effects in this partially drained case. The distribution of the effective pressure, $p^{\prime}$, is depicted at time $\mathrm{t}=0.15 \mathrm{~s}$. for both $\boldsymbol{u}-\boldsymbol{w}-p_{w}$ and $\boldsymbol{u}-\theta-\boldsymbol{w}-p_{w}$ formulations. Locking-induced numerical pollution that appears when the p-wave is traveling without stabilization is eliminated with the proposed technique (see Figure 11 . This volumetric locking arising from the effective response of the medium does not affect much motion at Point A in the free surface Figure 12 but it is very visible in the spurious mean stress oscillations that are recorded at point B (in Figure 12 b observed at two consecutive elements just above and below B).

For the undrained conditions $\left(k=10^{-10} \mathrm{~m} / \mathrm{s}\right)$ of this simulation the unstabilized element is severely unstable. As shown in Figure 13 large pore pressure oscillations are widespread across the domain, irrespective of location. The benefits of stabilization are highly significant in this case. Rayleigh-wave motion at A, Figure 14 , shows smaller damping (for the same time step and time-integration algorithm) using DME. The unacceptable performance of the non-stabilized solution is much clearer on the pore pressure wave at B, whereas, again, PPP stabilization does show some minor high frequency oscillation. 


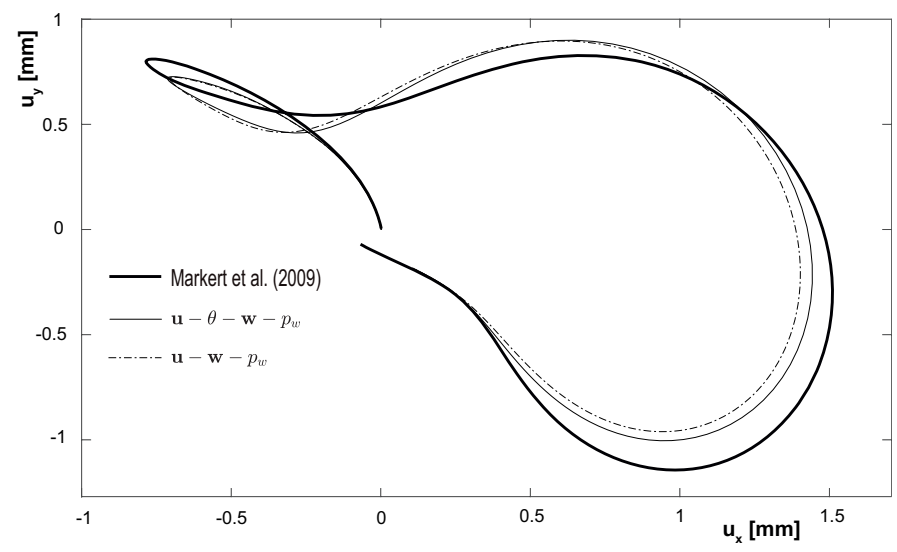

(a)

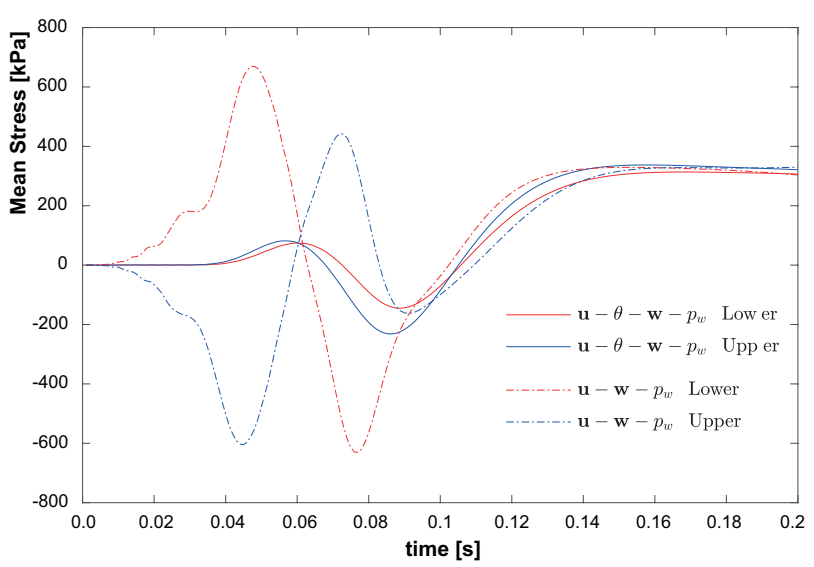

(b)

FIGURE 12 2D wave propagation in partially drained conditions. Comparison between formulations: $\boldsymbol{u}-\boldsymbol{w}-p_{w}$ and $\boldsymbol{u}-\theta-$ $\boldsymbol{w}-p_{w}$ : (a) In-plane motion of point A, and (b) mean stress distribution evolution at point B.

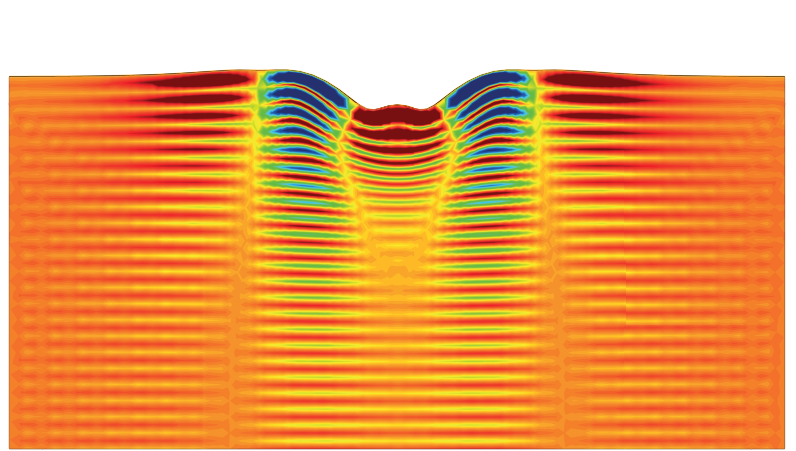

(a)

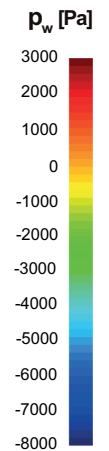

$-8000$

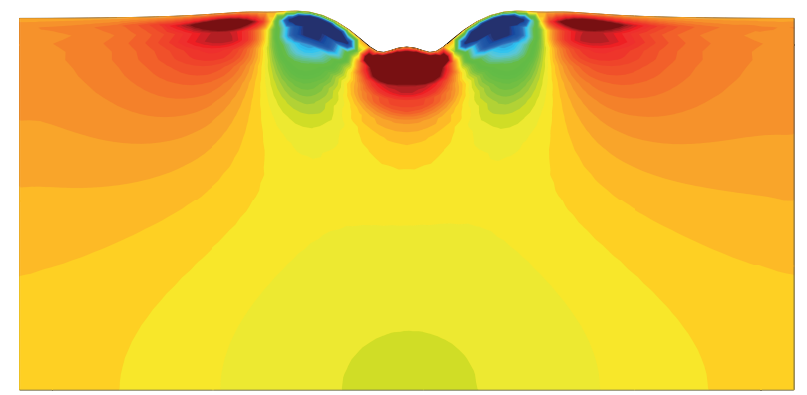

(b)

FIGURE $132 \mathrm{D}$ wave propagation in practically undrained conditions. Solution at $t=0.05 \mathrm{~s}$. Without stabilization of the mass balance equation, (a), and with stabilization of the mass balance equation, (b). (Contour displacements magnified x 250).

\section{5 | CONCLUSIONS}

This work presents an original approach for the numerical solution of the Biot equations that describe dynamic solid-fluid interaction, in which a formulation $\boldsymbol{u}-\boldsymbol{w}-p_{w}$ is employed. This formulation covers all drainage conditions, including those developing under high frequency or impact loading. The finite element approximation uses only linear shape functions, simplifying the implementation and reducing the computational load.

Several stabilization techniques, (polynomial pressure projection; divergence of momentum; mixed reformulation of field equations), are implemented to avoid spurious water pressure oscillations and volumetric locking of the low order elements employed. They all seem to perform well, as illustrated by several numerical examples for which reference solutions were available. The examples also illustrate that, with this formulation, numerical stabilization is required even in partly drained cases.

Although in the illustrative examples presented small strain conditions are prevalent, the formulation has been developed more generally, for the large strain regime that is relevant in many geotechnical applications. The formulation is presented in some detail, including a procedure to estimate stabilization parameter for the PPP stabilization term and, for the first time for large strain full Biot case, the set of consistent linearized equations. Further work will develop relevant solid contact algorithms and use more realistic constitutive models to test this formulation in geotechnical applications within the G-PFEM code. 


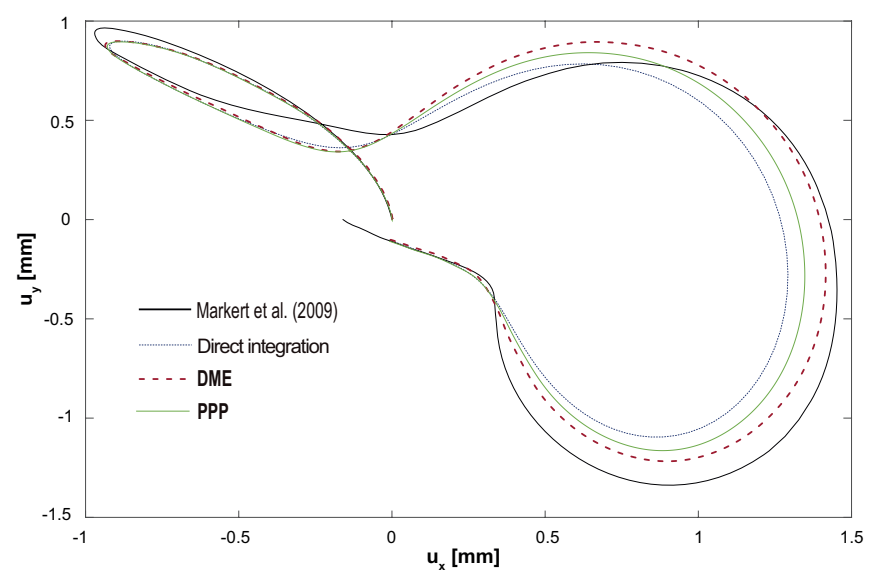

(a)

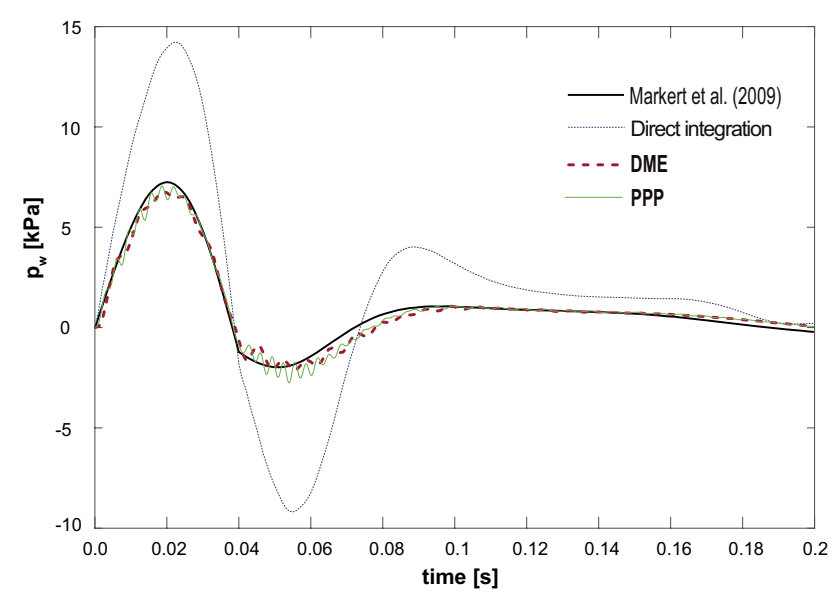

(b)

FIGURE 14 2D wave propagation in practically undrained conditions: (a) In-plane motion at point A, and (b) pore pressure evolution at point $\mathrm{B}$.

\section{ACKNOWLEDGMENTS}

This work has been supported by the Research Directorate of Spain (DGICYT) through research grants BIA2011-27217 and BIA2014-59467-R.

How to cite this article: Lluís Monforte, Pedro Navas, Josep Maria Carbonell, Marcos Arroyo and Antonio Gens (2018), Low order stabilized finite element for the full Biot formulation in Soil Mechanics at finite strains, I.J. Num. and Anal. Meth. Geomechanics., 2018;00:1-6.

\section{APPENDIX}

\section{A CONSISTENT LINEARIZATION}

As the linearization is referred to the undeformed domain, $\Omega_{0}$, since it is time independent, it is necessary to move Eqs. 42 . 44. to the reference configuration. From the transport theorems we know that $d \Omega=J d \Omega_{0}$ and $d \gamma=J \boldsymbol{F}^{-T} d \gamma_{0}$ and the Piola transformation states that $\operatorname{Div}(\boldsymbol{u})=J \operatorname{div}(\boldsymbol{u})$ (See $\sqrt{5859}$ for more information). Starting from these points, the equations to be linearized yield

$$
\begin{gathered}
-\alpha_{1} \int_{\Omega_{0}} \delta \boldsymbol{u} \cdot\left[\rho_{0} \boldsymbol{u}+J \rho_{w} \boldsymbol{w}\right] \mathrm{d} \Omega_{0}-\int_{\Omega_{0}} \boldsymbol{\tau}^{\prime}: \operatorname{grad} \delta \boldsymbol{u} \mathrm{d} \Omega_{0}+\int_{\Omega_{0}} J p_{w} \boldsymbol{I}: \operatorname{grad} \delta \boldsymbol{u} \mathrm{d} \Omega_{0}+\int_{\Omega_{0}} \delta \boldsymbol{u} \cdot \rho_{0} \boldsymbol{g} \mathrm{d} \Omega_{0}+\int_{\Gamma_{t 0}} \overline{\boldsymbol{T}} \cdot \delta \boldsymbol{u} \mathrm{d} \gamma_{0}=0 \\
-\int_{\Omega_{0}} J \operatorname{grad}\left(p_{w}\right) \cdot \delta \boldsymbol{w} \mathrm{d} \Omega_{0}-\alpha_{4} \int_{\Omega_{0}} J \boldsymbol{w} \cdot \mathbf{k}^{-1} \cdot \delta \boldsymbol{w} \mathrm{d} \Omega_{0}-\alpha_{1} \int_{\Omega_{0}} J \rho_{w}\left[\boldsymbol{u}+\frac{\boldsymbol{w}}{n}\right] \cdot \delta \boldsymbol{w} \mathrm{d} \Omega_{0}+\int_{\Omega_{0}} J \rho_{w} \boldsymbol{g} \cdot \delta \boldsymbol{w} \mathrm{d} \Omega_{0}=0 \\
\alpha_{4} \int_{\Omega_{0}} \operatorname{Div}(\boldsymbol{u}) \cdot \delta p_{w} \mathrm{~d} \Omega_{0}+\alpha_{4} \int_{\Omega_{0}} \operatorname{Div}(\boldsymbol{w}) \cdot \delta p_{w} \mathrm{~d} \Omega_{0}+\alpha_{4} \int_{\Omega_{0}} J \frac{p_{w}}{Q} \cdot \delta p_{w} \mathrm{~d} \Omega_{0}=0
\end{gathered}
$$


where $\boldsymbol{\tau}^{\prime}$ is the effective Kirchhoff stress tensor and $\overline{\boldsymbol{T}}$ is the traction vectors of solid phase computed respect the undeformed configuration. Following, the linearization of some useful terms is carried out against $\Delta \boldsymbol{u}$ (see ${ }^{27}$ for details):

(From ${ }^{60}$ )

$$
D_{u}[J]=J \operatorname{div}(\Delta u)
$$

$($ See also $\sqrt{59})$

$$
\begin{gathered}
D_{u}[n]=D_{u}\left[1-\frac{1-n_{0}}{J}\right]=(1-n) \operatorname{div}(\Delta \boldsymbol{u}) \\
D_{u}\left[\frac{1}{Q}\right]=D_{u}\left[\frac{n}{K_{w}}\right]=\frac{1}{K_{w}} D_{u}[n]=\frac{1-n}{K_{w}} \operatorname{div}(\Delta \boldsymbol{u}) \\
D_{u}[k]=(1-n) \frac{\partial k}{\partial n} \operatorname{div}(\Delta \boldsymbol{u})
\end{gathered}
$$

As the reference density is defined as

$$
D_{u}\left[J \frac{\rho_{w}}{n}\right]=\frac{\rho_{w}}{n} J \operatorname{div}(\Delta \boldsymbol{u})-J \frac{\rho_{w}}{n^{2}}(1-n) \operatorname{div}(\Delta \boldsymbol{u})=J \frac{\rho_{w}}{n} \frac{2 n-1}{n} \operatorname{div}(\Delta \boldsymbol{u})
$$

the linearization of the density yields:

$$
\rho_{0}=J \rho=J n \rho_{w}+J(1-n) \rho_{s}=J \rho_{w}-\left(1-n_{0}\right) \rho_{w}+\left(1-n_{0}\right) \rho_{s},
$$

From tensor analysis ${ }^{61}$ we determine that:

$$
D_{u}\left[\rho_{0}\right]=D_{u}\left[J \rho_{w}\right]=J \rho_{w} \operatorname{div}(\Delta u)
$$

$$
D_{u}[\operatorname{grad}(\delta \boldsymbol{u})]=D_{u}\left[\operatorname{Grad}(\delta \boldsymbol{u}) \boldsymbol{F}^{-1}\right]=\operatorname{Grad}\left(D_{u}[\boldsymbol{u}]\right) \boldsymbol{F}^{-1}+\operatorname{Grad}(\delta \boldsymbol{u}) D_{u}\left[\boldsymbol{F}^{-1}\right]=-\operatorname{grad}(\delta \boldsymbol{u}) \operatorname{grad}(\Delta \boldsymbol{u}),
$$

which is analogous for $D_{u}\left[\operatorname{grad}\left(p_{w}\right)\right]$, yielding: $-\operatorname{grad}\left(p_{w}\right) \operatorname{grad}(\Delta \boldsymbol{u})$. Also, the following term, either for $\operatorname{Div}(\boldsymbol{u})$ or $\operatorname{Div}(\boldsymbol{w})$ is linearized as:

$$
D_{u}[\operatorname{Div}(\boldsymbol{u})]=D_{u}[\boldsymbol{I}: \operatorname{Grad}(\boldsymbol{u})]=\boldsymbol{I}: \boldsymbol{D}_{u}[\operatorname{Grad}(\boldsymbol{u})]=\boldsymbol{I}: \operatorname{Grad}\left(D_{u}[\boldsymbol{u}]\right)=\boldsymbol{I}: \operatorname{Grad}(\Delta \boldsymbol{u})=\operatorname{Div}(\Delta \boldsymbol{u})
$$

The linearization will be stated for the weak form with respect to the reference configuration. Hereinafter the linearization of the main terms that upon the deformation field are presented. In the following equations superscripts represent the different terms of the Eqs. A1 A3):

- $D G_{L M S} \cdot \Delta u$ :

$$
\begin{gathered}
D G_{L M S}^{1} \cdot \Delta \boldsymbol{u}=D_{u}\left[\rho_{0} \boldsymbol{u}+J \rho_{w} \boldsymbol{w}\right] \cdot \delta \boldsymbol{u}=J\left[\rho \Delta \boldsymbol{u}+\rho_{w} \operatorname{div}(\Delta \boldsymbol{u})(\boldsymbol{u}+\boldsymbol{w})\right] \cdot \delta \boldsymbol{u} \\
D G_{L M S}^{2} \cdot \Delta \boldsymbol{u}=D_{u}\left[\boldsymbol{\tau}^{\prime}: \operatorname{grad}(\delta \boldsymbol{u})\right]=\operatorname{grad}(\Delta \boldsymbol{u}) \boldsymbol{\tau}^{\prime}: \operatorname{grad}(\delta \boldsymbol{u})+J \operatorname{grad}(\Delta \boldsymbol{u}): \boldsymbol{C}^{e p}: \operatorname{grad}(\delta \boldsymbol{u})
\end{gathered}
$$

where $\boldsymbol{C}^{e p}$ is the material elasto-plastic constitutive tangent operator. This linearization is widely developed in literature ${ }^{35}$. Also the linearization of the following term is well-known:

$$
\begin{aligned}
D G_{L M S}^{3} \cdot \Delta \boldsymbol{u} & =D_{u}\left[J p_{w} \boldsymbol{I}: \operatorname{grad}(\delta \boldsymbol{u})\right]=D_{u}[J] p_{w} \boldsymbol{I}: \operatorname{grad}(\delta \boldsymbol{u})+J p_{w} \boldsymbol{I}: D_{u}[\operatorname{grad}(\delta \boldsymbol{u})] \\
& =J p_{w} \operatorname{grad}(\delta \boldsymbol{u}):\left[\operatorname{div}(\Delta \boldsymbol{u}) \boldsymbol{I}-\operatorname{grad}^{T}(\Delta \boldsymbol{u})\right]
\end{aligned}
$$

- $D G_{L M S} \cdot \Delta \boldsymbol{w}:$

$$
D G_{L M S}^{1} \cdot \Delta \boldsymbol{w}=D_{w}\left[\rho_{0} \boldsymbol{u}+J \rho_{w} \boldsymbol{w}\right]=J \rho_{w} \Delta \boldsymbol{w}
$$

- $D G_{L M S} \cdot \Delta p_{w}$ :

$$
D G_{L M S}^{3} \cdot \Delta p_{w}=D_{p_{w}}\left[J p_{w} \boldsymbol{I}: \operatorname{grad}(\delta \boldsymbol{u})\right]=J \Delta p_{w} \boldsymbol{I}: \operatorname{grad}(\delta \boldsymbol{u})
$$


- $D G_{L M W} \cdot \Delta u$ :

$$
\begin{aligned}
D G_{L M W}^{1} \cdot \Delta \boldsymbol{u} & =D_{u}\left[J \operatorname{grad}\left(p_{w}\right) \cdot \delta \boldsymbol{w}\right]=D_{u}[J] \operatorname{grad}\left(p_{w}\right) \cdot \delta \boldsymbol{w}+J D_{u}\left[\operatorname{grad}\left(p_{w}\right)\right] \cdot \delta \boldsymbol{w} \\
& =\boldsymbol{J}\left[\operatorname{div}(\Delta \boldsymbol{u}) \operatorname{grad}\left(p_{w}\right)-\operatorname{grad}^{T}(\Delta \boldsymbol{u}) \operatorname{grad}\left(p_{w}\right)\right] \cdot \delta \boldsymbol{w}
\end{aligned}
$$

- $D G_{L M W} \cdot \Delta \boldsymbol{w}$ :

$$
\begin{gathered}
D G_{L M W}^{2} \cdot \Delta \boldsymbol{w}=D_{w}\left[J \boldsymbol{w} \cdot \mathbf{k}^{-1} \cdot \delta \boldsymbol{w}\right]=\frac{J}{k}\left[\operatorname{div}(\Delta \boldsymbol{u})\left(1-\frac{1-n}{k} \frac{\partial k}{\partial n}\right) \boldsymbol{w}\right] \cdot \delta \boldsymbol{w} \\
D G_{L M W}^{3} \cdot \Delta \boldsymbol{w}=D_{w}\left[J \rho_{w}\left(\boldsymbol{u}+\frac{\boldsymbol{w}}{n}\right) \cdot \delta \boldsymbol{w}\right]=\frac{J \rho_{w}}{n}\left[\frac{2 n-1}{n} \operatorname{div}(\Delta \boldsymbol{u}) \boldsymbol{w}\right] \cdot \delta \boldsymbol{w}
\end{gathered}
$$

- $D G_{L M W} \cdot \Delta p_{w}$ :

$$
D G_{L M W}^{1} \cdot \Delta p_{w}=D_{p_{w}}\left[J \operatorname{grad}\left(p_{w}\right) \cdot \delta \boldsymbol{w}\right]=J \operatorname{grad}\left(\Delta p_{w}\right) \cdot \delta \boldsymbol{w}
$$

- $D G_{M B E} \cdot \Delta u$ :

$$
\begin{aligned}
D G_{M B E}^{3} \cdot \Delta \boldsymbol{u} & =D_{u}\left[J \frac{p_{w}}{Q} \cdot \delta p_{w}\right]=D_{u}[J] \frac{p_{w}}{Q} \cdot \delta p_{w}+J p_{w} D_{u}\left[\frac{1}{Q}\right] \cdot \delta p_{w} \\
& =J p_{w}\left[\frac{n}{K_{w}}+\frac{1-n}{K_{w}}\right] \operatorname{div}(\Delta \boldsymbol{u}) \cdot \delta p_{w}=J \frac{p_{w}}{n Q} \operatorname{div}(\Delta \boldsymbol{u}) \cdot \delta p_{w}
\end{aligned}
$$

Taking into account these and the rest of analogous terms, the final linearization of Eqs. A1 A3 gives the following results:

$$
\begin{aligned}
& -\alpha_{1} \int_{\Omega}\left[\rho \Delta \boldsymbol{u}+\rho_{w} \Delta \boldsymbol{w}+\rho_{w} \operatorname{div}(\Delta \boldsymbol{u})(\boldsymbol{u}+\boldsymbol{w})\right] \cdot \delta \boldsymbol{u} \mathrm{d} \Omega-\int_{\Omega}\left(\operatorname{grad}(\delta \boldsymbol{u}): \boldsymbol{c}^{e p}: \operatorname{grad}(\Delta \boldsymbol{u})+\boldsymbol{\sigma}^{\prime}: \operatorname{grad}^{T}(\delta \boldsymbol{u}) \operatorname{grad}(\Delta \boldsymbol{u})\right) \mathrm{d} \Omega \\
& +\int_{\Omega}\left(\Delta p_{w} \operatorname{div}(\delta \boldsymbol{u})+p_{w} \operatorname{grad}(\delta \boldsymbol{u}):\left[\operatorname{div}(\Delta \boldsymbol{u}) \boldsymbol{I}-\operatorname{grad}^{T}(\Delta \boldsymbol{u})\right]\right) \mathrm{d} \Omega+\int_{\Omega} \rho_{w} \boldsymbol{g} \operatorname{div}(\Delta \boldsymbol{u}) \cdot \delta \boldsymbol{u} \mathrm{d} \Omega \\
& -\int_{\Omega}\left[\operatorname{grad}\left(\Delta p_{w}\right)+\operatorname{div}(\Delta \boldsymbol{u}) \operatorname{grad}\left(p_{w}\right)-\operatorname{grad}^{T}(\Delta \boldsymbol{u}) \operatorname{grad}\left(p_{w}\right)\right] \cdot \delta \boldsymbol{w} \mathrm{d} \Omega-\alpha_{4} \int_{\Omega}\left[\Delta \boldsymbol{w}+\operatorname{div}(\Delta \boldsymbol{u}) \frac{k-1+n}{k} \frac{\partial k}{\partial n} \boldsymbol{w}\right] \cdot \mathbf{k}^{-1} \cdot \delta \boldsymbol{w} \mathrm{d} \Omega \\
& -\alpha_{1} \int_{B} \frac{\rho_{w}}{n}\left[\Delta \boldsymbol{w}+\frac{2 n-1}{n} \operatorname{div}(\Delta \boldsymbol{u}) \boldsymbol{w}\right] \cdot \delta \boldsymbol{w} \mathrm{d} \Omega-\alpha_{1} \int_{\Omega} \rho_{w} \cdot[\Delta \boldsymbol{u}+\operatorname{div}(\Delta \boldsymbol{u}) \boldsymbol{u}] \cdot \delta \boldsymbol{w} \mathrm{d} \Omega+\int_{\Omega} \rho_{w} \boldsymbol{g} \operatorname{div}(\Delta \boldsymbol{u}) \cdot \delta \boldsymbol{w} \mathrm{d} \Omega \\
& (\mathrm{A} 24) \\
& \alpha_{4} \int_{\Omega} \operatorname{div}(\Delta \boldsymbol{u}) \cdot \delta p_{w} \mathrm{~d} \Omega+\alpha_{4} \int_{\Omega} \operatorname{div}(\Delta \boldsymbol{w}) \cdot \delta p_{w} \mathrm{~d} \Omega+\alpha_{4} \int_{\Omega}\left[\frac{\Delta p_{w}}{Q}+\frac{p_{w}}{n Q} \operatorname{div}(\Delta \boldsymbol{u})\right] \cdot \delta p_{w} \mathrm{~d} \Omega
\end{aligned}
$$

\section{B ESTIMATION OF THE STABILIZATION PARAMETER FOR THE PPP TECHNIQUE}

In stabilization techniques a free numerical parameter appears, which is related to the size of the element, the time increment and material constitutive parameters 34 . However, obtaining estimates for this parameter is rather complex in any particular case. In this work, to estimate the stabilization factor the same technique that was proposed by Cui et al ${ }^{40}$ to obtain the critical time-step for implicit methods is used.

The rational of the method proposed by Cui et ${ }^{40}$ is quite simple and is based on the solution of the one-dimensional consolidation problem in the quasi-static regime. It is assumed that all the nodes of the mesh have the same initial water pressure; at one extreme of the mesh the water pressure is increased whereas the other extreme of the mesh has Neumann boundary conditions. In order to avoid oscillations on the computed water pressure, the solution has to fulfill two conditions: 
- The nodal water pressure should be greater than the initial one at every time step.

- At a given time, the water pressure field should monotonically increase or decrease along the bar.

Analyzing the solution at the first time step for linear elements using a completely implicit time marching scheme, the authors obtained that the critical time step should be:

$$
\Delta t \geq \frac{h^{2}}{6 c_{v}}
$$

where $c_{v}=\frac{M \kappa}{g \rho_{w}}$ is the coefficient of consolidation, $M$ is the constrained modulus and $h$ the element size. This time constraint is equivalent to the one presented by Vermeer and Verruijt ${ }^{39}$ using a similar method.

In the proposed methodology small strains, one dimensional conditions, null boundary loads and linear elasticity are assumed. Further assuming that the total stress is constant over time, the following constitutive equation can be written:

$$
\nabla \cdot \dot{\boldsymbol{u}}=\dot{\varepsilon}_{v}=\dot{\varepsilon}_{z}=\frac{\dot{\sigma}_{z}^{\prime}}{M}=\frac{\dot{\sigma}_{z}+\dot{p}_{w}}{M}=\frac{\dot{p}_{w}}{M}
$$

and additionally this hypothesis implies:

$$
\begin{gathered}
\dot{p}^{\prime}=\dot{p}+\dot{p}_{w}=\dot{p}_{w} \\
\dot{\sigma}_{x}^{\prime}=\dot{\sigma}_{x}+\dot{p}_{w}=\dot{p}_{w}
\end{gathered}
$$

Introducing Equation Eq.(B27) to the mass balance equation yields:

$$
-\nabla \cdot \dot{\boldsymbol{w}}=\nabla \cdot \dot{\boldsymbol{u}}+\frac{1}{Q} \dot{p}_{w}=\left[\frac{1}{Q}+\frac{1}{M}\right] \dot{p}_{w}
$$

In order to obtain a single balance equation in terms of the water pressure, let us first obtain the divergence of the linear momentum of the mixture and the fluid:

$$
\begin{aligned}
\nabla^{2} \boldsymbol{\sigma}^{\prime}-\nabla^{2} p_{w}-\rho \nabla \ddot{\boldsymbol{u}}-\rho_{w} \nabla \ddot{\boldsymbol{w}} & =0 \\
-\nabla^{2} p_{w}-\frac{1}{k} \nabla \dot{\boldsymbol{w}}-\rho_{w} \nabla \ddot{\boldsymbol{u}}-\frac{\rho_{w}}{n} \nabla \ddot{\boldsymbol{w}} & =0
\end{aligned}
$$

where the divergence of $\mathbf{g}$ and the densities are neglected.

Subtracting Equation (B32) multiplied by the porosity, $n$, to Equation (B31) renders:

$$
k \nabla^{2} p_{w}-\left[\frac{1}{Q}+\frac{1}{M}\right] \dot{p}_{w}-\frac{k}{n} \frac{\rho_{d}}{M} \ddot{p}_{w}=0
$$

where $\rho_{d}$ is the dry density. For quasi-static range, the problem becomes:

$$
k \frac{\partial^{2} p_{w}}{\partial x^{2}}-\frac{\dot{p}_{w}}{M^{\star}}=0
$$

where $\frac{1}{M^{\star}}=\frac{1}{O}+\frac{1}{M}$ has been introduced to simplify the notation.

The semi-discrete finite element equations for the problem defined in Equation $(\overline{\mathrm{B} 33})$ reads:

$$
\frac{k}{n} \frac{\rho_{d}}{M} \mathbf{M} \cdot \ddot{\mathbf{p}}_{w}^{n+1}+\frac{1}{M^{\star}} \mathbf{M} \cdot \dot{\mathbf{p}}_{w}^{n+1}+\mathbf{M}^{s} \cdot \dot{\mathbf{p}}_{w}^{n+1}+\mathbf{H} \cdot \mathbf{p}_{w}^{n+1}=\mathbf{0}
$$

where $\mathbf{p}_{w}^{n+1}$ are the nodal values of the water pressures at $t_{n+1}, \mathbf{M}$ is the mass matrix, $\mathbf{M}^{s}$ is the matrix due to the stabilization technique and $\mathbf{H}$ is the permeability matrix. The elemental matrices are given by:

$$
\mathbf{M}_{e}=\frac{h}{6}\left[\begin{array}{ll}
2 & 1 \\
1 & 2
\end{array}\right] \quad \mathbf{M}_{e}^{s}=\tau \frac{h}{12}\left[\begin{array}{cc}
1 & -1 \\
-1 & 1
\end{array}\right] \quad \mathbf{H}_{e}=\frac{k}{h}\left[\begin{array}{cc}
1 & -1 \\
-1 & 1
\end{array}\right]
$$

where $h$ is the nodal spacing.

The same Newmark time-integration scheme is used for this simplified equation; thus, at the first time increment, the linear system of equations to be solved reads:

$$
\left(-\alpha_{1} \frac{k}{n} \frac{\rho_{d}}{M} \mathbf{M}+\alpha_{4}\left[\mathbf{M}^{s}+\frac{1}{M^{\star}} \mathbf{M}\right]+\mathbf{H}\right) \cdot \mathbf{p}_{w}^{1}=\mathbf{0}
$$


TABLE B1 Stabilization factors proposed for the PPP technique.

\begin{tabular}{ll}
\hline Reference & Stabilization factor, $\tau$ \\
\hline White \& Borja ${ }^{17}$ & $\frac{1}{2 G}=\frac{1-\nu}{1-2 v} \frac{1}{M}$ \\
Sun et al ${ }^{18}$ & $\left(\frac{2}{M}-\frac{6 k \Delta t}{h^{2}}\right)\left(\frac{1}{2}+\frac{1}{2} \tanh \left(2-\frac{12 k M \Delta t}{h^{2}}\right)\right)$ \\
Li \& Wei ${ }^{62}$ & $\frac{3}{M}+\frac{2}{Q}$ \\
This work & $\max \left(\frac{2}{M}+\frac{2}{Q}-\frac{\beta}{\gamma} \frac{12 \Delta t k}{h^{2}}, 0\right)$ \\
\hline
\end{tabular}

The last equation of this system of equations, Equation (B37), relates the value of the water pressure at the last node of the oedometer (the one where null Neumann boundary conditions are applied) and its neighbor:

$$
\left(\alpha_{4}\left[\frac{h}{6 M^{\star}}-\frac{\tau h}{12}\right]-\frac{k}{h}+\alpha_{1} \frac{h k \rho_{d}}{6 n M}\right)\left(\Delta p_{w}\right)_{m-1}^{1}+\left(\alpha_{4}\left[\frac{2 h}{6 M^{\star}}+\frac{\tau h}{12}\right]+\frac{k}{h}+\alpha_{1} \frac{2 h k \rho_{d}}{6 n M}\right)\left(\Delta p_{w}\right)_{m}^{1}=0
$$

where $\left(\Delta p_{w}\right)_{m}^{1}$ is the excess water pressure (the current water pressure minus the initial value) at the last node of the bar at time $\Delta t$ whereas $\left(\Delta p_{w}\right)_{m-1}^{1}$ is the node adjacent to the last one.

The two aforementioned requirements that the solution must fulfill ${ }^{40}$ may be announced as:

$$
1 \geq \frac{\left(\Delta p_{w}\right)_{m}^{1}}{\left(\Delta p_{w}\right)_{m-1}^{1}}=\frac{-\alpha_{4}\left[\frac{h}{6 M^{\star}}-\frac{\tau h}{12}\right]+\frac{k}{h}-\alpha_{1} \frac{h k \rho_{d}}{6 n M}}{\alpha_{4}\left[\frac{2 h}{6 M^{\star}}+\frac{\tau h}{12}\right]+\frac{k}{h}+\alpha_{1} \frac{2 h k \rho_{d}}{6 n M}} \geq 0
$$

The first inequality do not pose any restriction on the value of $\tau$. Meanwhile, the second inequality is relevant; since the denominator is always positive, the numerator should also be larger or equal to zero. Then, after some manipulation it yields:

$$
\tau \geq \frac{2}{M}\left(1+\frac{1}{\gamma \Delta t} \frac{k \rho_{d}}{n}\right)+\frac{2}{Q}-\frac{\beta \Delta t}{\gamma} \frac{12 k}{h^{2}},
$$

For a quasi-static problem, neglecting dynamic terms, the stabilization factor to be used is obtained as:

$$
\tau \geq \frac{2}{M}+\frac{2}{Q}-\frac{\beta \Delta t}{\gamma} \frac{12 k}{h^{2}}
$$

Since the stabilization factor should be positive, the following approximation is proposed:

$$
\tau= \begin{cases}\frac{2}{M}+\frac{2}{Q}-\frac{\beta}{\gamma} \frac{12 \Delta t k}{h^{2}} & \text { if } \Delta t \leq \frac{\gamma}{\beta} \frac{h^{2}}{6}\left(\frac{1}{c_{v}}+\frac{1}{k Q}\right) \\ 0 & \text { other cases }\end{cases}
$$

Table B1 presents some of the stabilization factors proposed in the literature for the PPP technique for $\mathbf{u}-p_{w}$ elements using a completely implicit time marching scheme, assuming linear elasticity and neglecting dynamic effects. It is worth noting that the factor proposed here has a similar expression to the one found by Sun et al ${ }^{[18}$ using a more complex technique ${ }^{63}$; in fact, Sun et al ${ }^{18}$ include the term related to tanh to limit the overdiffusion. It is also remarkable the similitude with the stabilization factor proposed by Li \& Wei ${ }^{62}$ ? they do not have the negative term and the inverse of the constrained modulus is multiplied by 3 instead of 2 . The stabilization factor proposed by White and Borja ${ }^{17}$ depends on the shear modulus instead of the constrained modulus.

In order to assess the stabilization technique, the one-dimensional oedometer is computed and the solution is compared with that of Terzaghi. The height is $1 \mathrm{~m}$, the coefficient of consolidation is $c_{v}=1 \mathrm{~m}^{2} / \mathrm{s}$, whereas the constrained modulus is $M=100$ $\mathrm{kPa}$. The Poisson's ratio has been fixed at $v=1 / 3$; this way, the proposal of White and Borja ${ }^{17}$ coincides with that of Sun et a ${ }^{18}$ and the one derived in this work once the term $k \Delta t / h^{2}$ tends to zero. However, it should be stressed that the proposal of White and Borja ${ }^{17}$ would render different results by assuming different Poisson's ratios.

Figure B1 the results of a convergence analysis, where the solution is computed using 500 time steps up to a final normalized time of $T=10^{-3}$. Two types of error norms are adopted in this study, namely, the $L^{2}$ norm

$$
\left\|p-p^{h}\right\|_{L^{2}}^{*}=\left(\frac{\int_{\Omega}\left(p(\mathbf{x})-p^{h}(\mathbf{x})\right)^{2} \mathrm{~d} \Omega}{\int_{\Omega} \mathrm{d} \Omega}\right)^{\frac{1}{2}}
$$




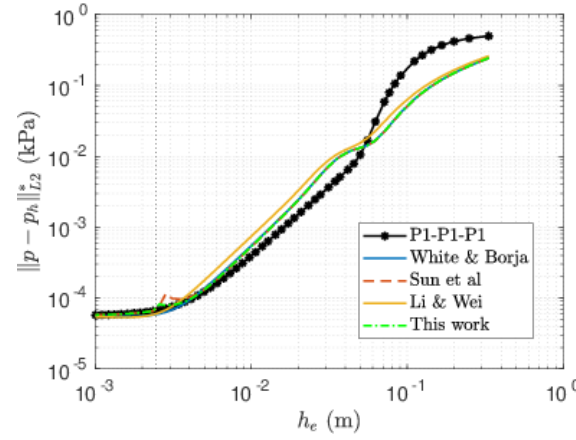

(a)

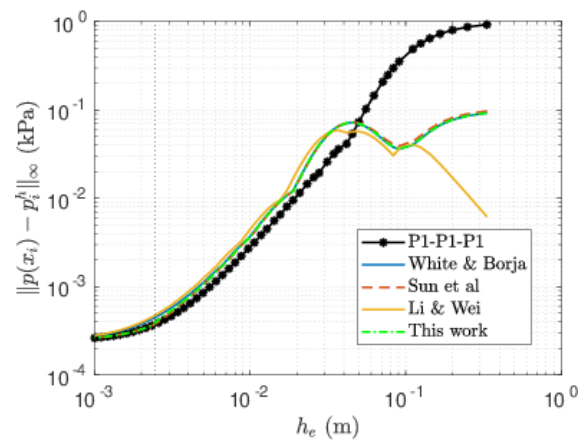

(d)

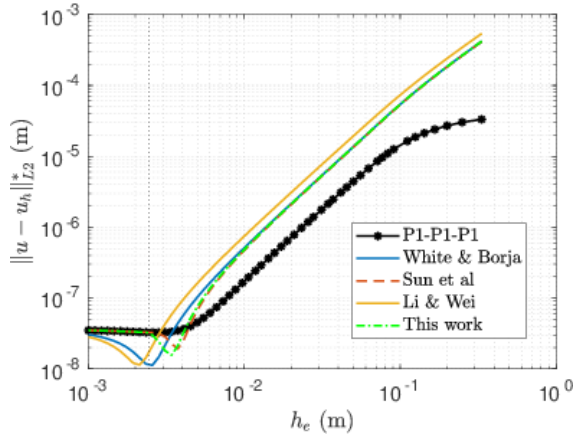

(b)

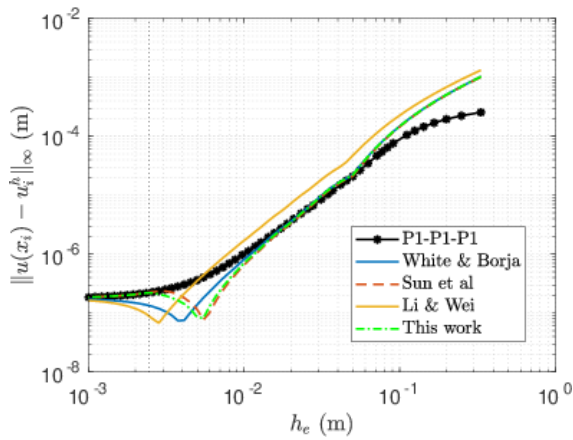

(e)

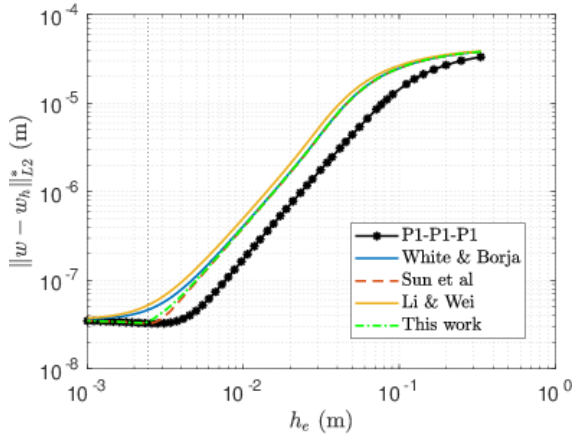

(c)

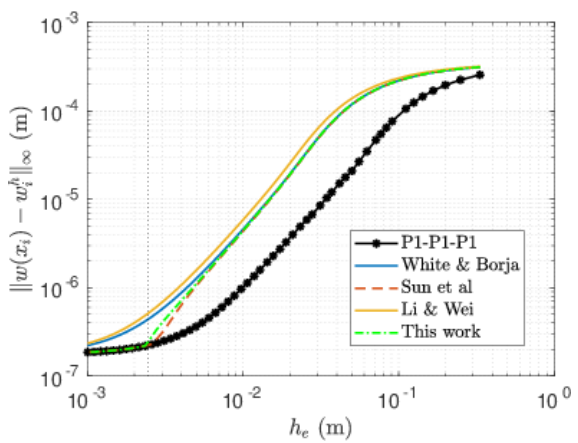

(f)

FIGURE B1 One-dimensional oedometer. Error norms for the unstabilized element (P1-P1-P1) and using several stabilization factors for the PPP technique proposed in the literature.

and the maximum nodal difference

$$
\left\|p-p^{h}\right\|_{\infty}=\max \left(p\left(\mathbf{x}_{\mathbf{i}}\right)-p^{h}\left(\mathbf{x}_{\mathbf{i}}\right)\right)
$$

As noted before, all the proposals for the value of the stabilization factor proposed in the literature are quite similar, see Table B1 and Equation (B42); it is not surprising then that the error norms for all variables show a similar trend irrespectively of the employed proposal. It should be noted that, with the exception of the water pressure nodal difference, the stabilization factor proposed by Li and Wei ${ }^{62}$, give consistently higher error norms. Additionally, these figures also include -indicated by a discontinuous vertical line- the element size at which the proposed stabilization factor is zero (see Equation (B42)); as such, at the left of this line the solution is computed with a null stabilization factor, whereas on the right the stabilization factor is larger than zero. Stabilization switch-off does not produce any undesirable effect.

\section{References}

1. Biot M. A.. Theory of propagation of elastic waves in a fluid-saturated porous solid. I. Low-Frequency range. Journal of the Acoustical Society of America. 1956;28(2):168-178.

2. Zienkiewicz O.C., Shiomi T.. Dynamic Behaviour of saturated porous media: The generalized Biot formulation and its numerical solution.. Int. J. Num. Anal. Geomech.. 1984;8:71-96.

3. Zienkiewicz O.C., Chan A.H.C., Pastor M., Schrefler B.A., Shiomi T.. Computational Geomechanics. UK: John Wiley; 1999.

4. Pastor M, Li T, Liu X, Zienkiewicz OC, Quecedo M. A fractional step algorithm allowing equal order of interpolation for coupled analysis of saturated soil problems. Mechanics of Cohesive-frictional Materials. 2000;5(7):511-534. 
5. Bathe K.J.. The inf-sup condition and its evaluation for mixed finite element methods. Computers and structures. 2010;79(2):243-245.

6. Oñate E. Derivation of stabilized equations for numerical solution of advective-diffusive transport and fluid flow problems. Computer Methods in Applied Mechanics and Engineering. 1998;151(1):233 - 265. Containing papers presented at the Symposium on Advances in Computational Mechanics.

7. Oñate E. Possibilities of finite calculus in computational mechanics. International Journal for Numerical Methods in Engineering. 2004;60(1):255-281.

8. Preisig M, Prévost J H. Stabilization procedures in coupled poromechanics problems: A critical assessment. International Journal for Numerical and Analytical Methods in Geomechanics. 2011;35(11):1207-1225.

9. Pouplana I, Oñate E. A FIC-based stabilized mixed finite element method with equal order interpolation for solid-pore fluid interaction problems. International Journal for Numerical and Analytical Methods in Geomechanics. 2017;41(1):110-134.

10. Pastor M, Li T, Liu X, Zienkiewicz O. C.. Stabilized low-order finite elements for failure and localization problems in undrained soils and foundations. Computer Methods in Applied Mechanics and Engineering. 1999;174(1-2):219-234.

11. Hughes T.J.R., Franca L.P., Balestra M.. A new finite element formulation for fluid dynamics, V. Circumventing the Babuška-Brezzi condition: A stable Petrov-Galerkin formulation of the Stokes problem accomodating equal order interpolation.. Computer Methods in Applied Mechanics and Engineering. 1986;59(1):85-99.

12. Oñate E, Idelsohn S R, Del Pin F, Aubry R. The particle finite element method - an overview. International Journal of Computational Methods. 2004;1(02):267-307.

13. Carbonell J M, Oñate E, Suárez B. Modelling of tunnelling processes and rock cutting tool wear with the particle finite element method. Computational Mechanics. 2013;52(3):607-629.

14. Monforte L, Arroyo M, Carbonell J M, Gens A. Numerical simulation of undrained insertion problems in geotechnical engineering with the particle finite element method (PFEM). Computers and Geotechnics. 2017;82:144-156.

15. Hafez M., Soliman M.. Numerical solution of the incompressible Navier-Stokes equations in primitive variables on unstaggered grids:368-379. American Institute of Aeronautics and Astronautics 1991.

16. Dohrmann C R, Bochev P B. A stabilized finite element method for the Stokes problem based on polynomial pressure projections. International Journal for Numerical Methods in Fluids. 2004;46(2):183-201.

17. White J A, Borja R I. Stabilized low-order finite elements for coupled solid-deformation/fluid-diffusion and their application to fault zone transients. Computer Methods in Applied Mechanics and Engineering. 2008;197(49):4353-4366.

18. Sun W., Ostien J. T., Salinger A. G.. A stabilized assumed deformation gradient finite element formulation for strongly coupled poromechanical simulations at finite strain. International Journal for Numerical and Analytical Methods in Geomechanics. 2013;37(16):2755-2788.

19. Pastor M, Li T, Fernández-Merodo J. A.. Stabilized finite elements for harmonic soil dynamics problems near the undrainedincompressible limit. Soil dynamics and Earthquake engineering. 1997;16(3):161-171.

20. Sun W., Chen Q., Ostien J. T.. Modeling the hydro-mechanical responses of strip and circular punch loadings on watersaturated collapsible geomaterials. Acta Geotechnica. 2014;9(5):903-934.

21. Monforte L, Carbonell J. M., Arroyo M., Gens A.. Performance of mixed formulations for the particle finite element method in soil mechanics problems. Computational Particle Mechanics. 2017;4(3):269-284.

22. Jeremić B., Cheng Z., Taiebat M., Dafalias Y.. Numerical simulation of fully saturated porous materials. International Journal for Numerical and Analytical Methods in Geomechanics. 2008;32:1635-1660.

23. Markert B, Heider Y, Ehlers W. Comparison of monolithic and splitting solution schemes for dynamic porous media problems. International Journal for Numerical Methods in Engineering. 2010;82(11):1341-1383. 
24. Sabetamal H, Nazem M, Sloan S W, Carter J P. Frictionless contact formulation for dynamic analysis of nonlinear saturated porous media based on the mortar method. International Journal for Numerical and Analytical Methods in Geomechanics. 2016;40(1):25-61.

25. Sabetamal H, Carter J P, Nazem M, Sloan S W. Coupled analysis of dynamically penetrating anchors. Computers and Geotechnics. 2016;77:26-44.

26. Navas P., Sanavia L., López-Querol S., Yu R.C.. Explicit meshfree solution for large deformation dynamic problems in saturated porous media.. Acta geotechnica. 2017;Published online:https://doi.org/10.1007/s11440-017-0612-7.

27. Navas P., Sanavia L., López-Querol S., Yu R.C.. u-w formulation for dynamic problems in large deformation regime solved through an implicit meshfree scheme.. Computational mechanics. 2017;Published online:https://doi.org/10.1007/s00466017-1524-y.

28. Idelsohn S.R., Oñate E., Pin F. Del. The particle finite element method: a powerful tool to solve incompressible flows with free-surfaces and breaking waves. International Journal for Numerical Methods in Engineering. 2004;61(7):964-989.

29. Monforte L., Arroyo M., Carbonell J. M., Gens A.. Coupled effective stress analysis of insertion problems in geotechnics with the Particle Finite Element Method. Computers and Geotechnics. 2018;101:114-129.

30. Lewis R.W., Schrefler B.A.. The finite element method in the static and dynamic deformation and consolidation of porous media. John Wiley \& Sons Ltd.; 1998.

31. Ferronato M., Castelletto N., Gambolati G.. A fully coupled 3-D mixed finite element model of Biot consolidation. Journal of Computational Physics. 2010;229(12):4813-4830.

32. Castelletto Nicola, White Joshua A., Ferronato Massimiliano. Scalable algorithms for three-field mixed finite element coupled poromechanics. Journal of Computational Physics. 2016;327:894 - 918.

33. Lotfian Z., Sivaselvan M.V.. Mixed finite element formulation for dynamics of porous media. International Journal for Numerical Methods in Engineering. ;115(2):141-171.

34. Zienkiewicz O.C., Taylor R.L.. The finite element method. Volume 1: Basic formulation and linear problems. London: McGraw-Hill; 1994.

35. Wriggers P.. Nonlinear Finite Element Methods. Springer; 2008.

36. Kontoe S., Zdravkovic L., Potts D.M.. An assessment of time integration schemes for dynamic geotechnical problems. Computers and Geotechnics. 2008;35:253-264.

37. Pastor M., Zienkiewicz O.C., Li T., Xiaoqing L., Huang M.. Stabilized Finite Elements with Equal Order of Interpolation for Soil Dynamics Problems.. Archives of Computational Methods in Engineering. 1999;6(1):3-33.

38. Hong Qingguo, Kraus Johannes. Parameter-robust stability of classical three-field formulation of Biot's consolidation model. arXiv preprint arXiv:1706.00724. 2017;.

39. Vermeer PA, Verruijt A. An accuracy condition for consolidation by finite elements. International Journal for Numerical and Analytical Methods in Geomechanics. 1981;5(1):1-14.

40. Cui W., Gawecka K. A., Taborda D. M. G., Potts D. M., Zdravković L.. Time-step constraints in transient coupled finite element analysis. International Journal for Numerical Methods in Engineering. 2016;106(12):953-971. nme.5141.

41. Bochev P B, Dohrmann C R, Gunzburger M D. Stabilization of low-order mixed finite elements for the Stokes equations. SIAM Journal on Numerical Analysis. 2006;44(1):82-101.

42. Rodriguez J. M., Carbonell J. M., Cante J. C., Oliver J.. The particle finite element method (PFEM) in thermo-mechanical problems. International Journal for Numerical Methods in Engineering. 2016;107(9):733-785. nme.5186.

43. Brezzi F., Pitkaranta J. On the stabilization of finite element approximation of the Stokes problem.. Efficient solitions of elliptic problems, Notes on Numerical Fluid Mechanics. 1984;10:11-19. 
44. Zienkiewicz O.C., Huang M., Pastor M.. Computational soil dynamics - A new algorithm for drained and undrained conditions.. Computer Methods and Advances in Geomechanics. 1994;8:47-59.

45. Souza Neto E. A., Perić D, Dutko M, Owen D. R. J.. Design of simple low order finite elements for large strain analysis of nearly incompressible solids. International Journal of Solids and Structures. 1996;33(20-22):3277-3296.

46. Souza Neto E. A., Andrade Pires F. M., Owen D. R. J.. F-bar-based linear triangles and tetrahedra for finite strain analysis of nearly incompressible solids. Part I: formulation and benchmarking. International Journal for Numerical Methods in Engineering. 2005;62(3):353-383.

47. Dadvand P Oñate E. An object-oriented environment for developing finite element codes for multi-disciplinary applications. Arch Comput Methods Eng. 2010;17(3):253-297.

48. Zienkiewicz O.C., Chang C.T., Bettes P.. Drained, undrained, consolidating and dynamic behaviour assumptions in soils. Géotechnique. 1980;30(4):385-395.

49. Navas P., López-Querol S., Yu R.C., Li B.. B-bar based algorithm applied to meshfree numerical schemes to solve unconfined seepage problems through porous media. International Journal for Numerical and Analytical Methods in Geomechanics. 2016;40:962-984.

50. Navas P., Yu R.C., López-Querol S., Li B.. Dynamic consolidation problems in saturated soils solved through u-w formulation in a LME meshfree framework. Computers and Geotechnics. 2016;79:55-72.

51. Biot M. A.. General solutions of the equations of elasticity and consolidation for a porous material. Journal of Applied Mechanics. 1956;:91-96.

52. Verruijt A.. An introduction to soil dynamics. Delft, The Netherlands: Springer; 2010.

53. Carter J P, Sabetamal H, Nazem M, Sloan S W. One-dimensional test problems for dynamic consolidation. Acta Geotechnica. 2015;10:173-178.

54. De Boer R, Ehlers W, Liu Z. One-dimensional transient wave propagation in fluid-saturated incompressible porous media. Archive of Applied Mechanics. 1993;63:59-72.

55. Breuer S.. Quasi-static and dynamic behavior of saturated porous media with incompressible constituents.. Transport in Porous Media. 1999;34:285-303.

56. Gajo A., Saetta A., Vitaliani R.. Silent boundary conditions for wave propagation in saturated porous media. International Journal for Numerical and Analytical Methods in Geomechanics. 1996;20(4):253-273.

57. Maleki Javan M. R., Noorzad A., Latifi Namin M.. Three-dimensional nonlinear finite element analysis of pile groups in saturated porous media using a new transmitting boundary. International Journal for Numerical and Analytical Methods in Geomechanics. 2007;32(6):681-699.

58. Marsden J.E., Hughes T. J. R.. Mathematical Foundations of Elasticity. Prentice Hall Inc., Upper Saddle River, New Jersey.; 1983.

59. Sanavia L., Schrefler B.A., Steinmann P.. A formulation for an unsaturated porous medium undergoing large inelastic strains.. Computational Mechanics. 2002;28:137-151.

60. Hughes T. J. R., Pister K. S.. Consistent linearization in mechanics of solids and structures.. Computers and structures. 1978;8:391-391.

61. Hughes T.J.R., Hilber H.M.. Collocation, dissipation and overshoot for time integration schemes in structural dynamics. Earthquake Engineering and Structural Dynamics. 1978;6:99-117.

62. Li Wentao, Wei Changfu. Stabilized low-order finite elements for strongly coupled poromechanical problems. International Journal for Numerical Methods in Engineering. ;115(5):531-548. 
63. Harari I. Stability of semidiscrete formulations for parabolic problems at small time steps. Computer Methods in Applied Mechanics and Engineering. 2004;193(15):1491 - 1516. Recent Advances in Stabilized and Multiscale Finite Element Methods. 